\title{
Star formation in the intragroup medium and other diagnostics of the evolutionary stages of compact groups of galaxies ${ }^{\star}$
}

\author{
S. Torres-Flores ${ }^{1,2}$, C. Mendes de Oliveira ${ }^{1}$, D. F. de Mello ${ }^{3,4,5}$, P. Amram² ${ }^{2}$ H. Plana ${ }^{2,7}$, \\ B. Epinat ${ }^{6}$, and J. Iglesias-Páramo ${ }^{8}$ \\ 1 Universidade de São Paulo, Instituto de Astronomia, Geofísica e Ciências Atmosféricas, Departamento de Astronomia, \\ São Paulo, Brazil \\ e-mail: storres@astro.iag.usp.br \\ 2 Laboratoire d'Astrophysique de Marseille, OAMP, Université de Provence \& CNRS, 38 rue F. Joliot-Curie, \\ 13388 Marseille Cedex 13, France \\ 3 Observational Cosmology Laboratory, Code 665, Goddard Space Flight Center, Greenbelt, MD 20771, USA \\ 4 Catholic University of America, Washington, DC 20064, USA \\ 5 Johns Hopkins University, Baltimore, MD 21218, USA \\ ${ }^{6}$ Laboratoire d'Astrophysique de Toulouse-Tarbes, Université de Toulouse, CNRS, 14 avenue Edouard Belin, 31400 Toulouse, \\ France \\ ${ }^{7}$ Laboratorio de Astrofisica Teorica e Observacional, Universidade Estadual de Santa Cruz, Brazil \\ 8 Instituto de Astrofisica de Andalucia (CSIC), Camino Bajo de Huetor 50, 18008 Granada, Spain
}

Received 19 February 2009 / Accepted 12 August 2009

\section{ABSTRACT}

\begin{abstract}
Context. Compact groups of galaxies are entities that have high densities of galaxies and serve as laboratories to study galaxy interactions, intergalactic star formation and galaxy evolution.

Aims. The main goal of this study is to search for young objects in the intragroup medium of seven compact groups of galaxies: HCG 2, 7, 22, 23, 92, 100 and NGC 92 as well as to evaluate the stage of interaction of each group.

Methods. We used Fabry-Perot velocity fields and rotation curves together with GALEX NUV and FUV images and optical $R$-band and HI maps.

Results. (i) HCG 7 and HCG 23 are in early stages of interaction; (ii) HCG 2 and HCG 22 are mildly interacting; and (iii) HCG 92, HCG 100 and NGC 92 are in late stages of evolution. We find that all three evolved groups contain populations of young blue objects in the intragroup medium, consistent with ages $<100 \mathrm{Myr}$, of which several are younger than $<10$ Myr. We also report the discovery of a tidal dwarf galaxy candidate in the tail of NGC 92. These three groups, besides containing galaxies that have peculiar velocity fields, also show extended HI tails.

Conclusions. Our results indicate that the advanced stage of evolution of a group, together with the presence of intragroup HI clouds, may lead to star formation in the intragroup medium. A table containing all intergalactic HII regions and tidal dwarf galaxies confirmed to date is appended.
\end{abstract}

Key words. galaxies: evolution - galaxies: interactions - galaxies: intergalactic medium - galaxies: kinematics and dynamics

\section{Introduction}

It is well known that tidal interactions can alter galaxies' properties and, in particular, neutral gas can be plucked by tidal forces and develop tidal tails where young stellar clusters, HII regions, and tidal dwarf galaxies (TDGs) may form (e.g. de Mello et al. 2008a,b; Mendes de Oliveira et al. 2004; Ryan-Weber et al. 2004; Mendes de Oliveira et al. 2001; and Duc \& Mirabel 1998). However, the fate of these young systems remains unclear (de Mello et al. 2008b) and they can either (1) become independent entities growing by accreting more gas and forming more stars to be transformed in TDGs; (2) make star clusters that survive and migrate in the distant halos of their hosts or even become intergalactic objects; or (3) dissolve and not remain gravitationally bound, yielding only very sparse star streams.

* Based on observations collected at the European Southern Observatory, La Silla, Chile.
Hunsberger et al. (1996) suggested that at least one-third and perhaps more than one-half of all dwarf galaxies in compact groups are formed during galaxy interactions. Therefore, a considerable number of new stellar systems may have formed and may be linked to the evolutionary stage of the group. There are several diagnostics used in the literature to evaluate these stages. For instance, Coziol et al. (2004) developed a scheme to classify the evolutionary stage of compact groups of galaxies based on the morphologies of the members and their level of activity. Plana et al. (2003) and Amram et al. (2003), using 2D H $\alpha$ velocity fields, classified several of the Hickson compact groups in three classes: unevolved group, mildly interacting group, and a system in the final stage of evolution. Verdes-Montenegro et al. (2001), on the other hand, used the HI content of compact groups to propose an evolutionary sequence. In their classification, groups may show HI still attached to the individual members, in tidal tails or in a common HI envelope around all 
galaxies. In this context, HI and kinematic information from $2 \mathrm{D}$-velocity maps have proved to be the most useful tools to study the evolutionary stages of groups.

In this paper, we present the results of a multiwavelength campaign we have conducted using several instruments in order to properly evaluate the evolutionary stages of seven compact groups of galaxies. We report the discovery of young objects in the intragroup medium of compact groups and in the tidal tails of strongly interacting galaxies, which may have formed due to galaxy interactions, using GALEX/UV data, optical R-images, new Fabry Perot velocity maps and HI maps from the literature. UV images reveal important information regarding the age of the young objects while the velocity fields and rotation curves of the group members help to constrain the evolutionary stage of each compact group.

This paper is organized as follows: in Sect. 2 we present the data. In Sect. 3 we present the data analysis. In Sect. 4 we present the ultraviolet and Fabry Perot results. In Sect. 5 we discuss our results and in Sect. 6 we present our main conclusions.

\section{Data}

In the following sections we describe the data we obtained for a sample of seven compact groups of galaxies: HCG 2, HCG 7 , HCG 22, HCG 23, HCG 92, HCG 100 and NGC 92. These nearby groups (with velocities lower than $7000 \mathrm{~km} \mathrm{~s}^{-1}$ ) span a wide range of properties (density, isolation, spiral fraction and HI content. See Hickson 1982; Verdes Montenegro et al. 2001). In particular, isolated groups may evolve differently from groups which are parts of larger structures (such as HCG 23, Williams \& van Gorkom 1995). On the other hand, HCG 92 and HCG 100 are known to have HI emission in the intragroup medium (Verdes-Montenegro et al. 2001; and de Mello et al. 2008a, respectively) while NGC 92 has a common halo of HI around the whole group (Pompei et al. 2007) and the others have HI emission which is more concentrated in the galaxies. Therefore, the groups chosen are representative of the general compact group population. In Table 1, we summarize the main properties of this sample.

\subsection{Fabry-Perot data}

Observations of HCG 2, HCG 7, HCG 22 and NGC 92 were carried out with the European Southern Observatory $3.6 \mathrm{~m}$ telescope (ESO) in September 2000. The data were obtained with the Fabry-Perot instrument CIGALE using an Image Photon Counting System, for a field of view of 4 arcmin centered on each group. These data are described in detail in Torres-Flores et al. (2009). The scale of the images was $0.405^{\prime \prime} /$ pixel. The data were taken under non-photometric conditions and with a seeing of $1^{\prime \prime}$. No flux calibration was attempted. Data reduction was performed using software developed by Daigle et al. (2006; see also Epinat et al. 2008a). The data for HCG 100 was taken from Plana et al. (2003).

The output of the reduction package gave us 2D monochromatic, continuum and radial velocity maps, with a signal-tonoise ratio higher than 6 . Wavelength calibration was obtained by scanning the narrow Ne $6599 \AA$ line under the same conditions as the observations. The velocity sampling was $12 \mathrm{~km} \mathrm{~s}^{-1}$. For HCG 2, 7 and 22, the $\mathrm{OH}$ subtraction was performed by estimating a sky cube from the sky-dominated regions and subtracting it from the original cube. In the case of NGC 92, the $\mathrm{OH}$ was subtracted estimating the sky using the medium spectrum of the data cube. This method resulted in a better velocity field for NGC 92 than when the sky cube method was adopted.

\subsection{Ultraviolet data}

The UV images of the seven compact groups in this sample were obtained using the Galaxy Evolution Explorer (GALEX) satellite in the near ultraviolet (NUV $\lambda_{\text {eff }}=2271 \AA$ ) and far ultraviolet (FUV $\lambda_{\text {eff }}=1528 \AA$ ) bands (Cycle 1, program number 73 and 31 and public archival data). The images analyzed herein are the intensity maps with the background removed (given by the GALEX pipeline). FUV and NUV fluxes were calculated using Morrissey et al. (2005) $m_{\lambda}=-2.5 \log \left[F_{\lambda} / a_{\lambda}\right]+b_{\lambda}$, where $a_{\mathrm{FUV}}=1.4 \times$ $10^{-15} \mathrm{erg} \mathrm{s}^{-1} \mathrm{~cm}^{-2} \AA^{-1}, a_{\mathrm{NUV}}=2.06 \times 10^{-16} \mathrm{erg} \mathrm{s}^{-1} \mathrm{~cm}^{-2} \AA^{-1}$, $b_{\mathrm{FUV}}=18.82$ and $b_{\mathrm{NUV}}=20.08$ for FUV and NUV, respectively. Fluxes were multiplied by the effective filter bandpass $\left(\Delta \lambda_{\mathrm{FUV}}=\right.$ $269 \AA$ and $\left.\Delta \lambda_{\mathrm{NUV}}=616 \AA\right)$ to give units of erg s $\mathrm{cm}^{-1}$. The GALEX fields view are 1.28 and 1.24 in FUV and NUV respectively and the pixel scale is 1.5 arcsec pixel ${ }^{-1}$.

HCG 100 and HCG 92 were originally analyzed in de Mello et al. (2008a) ${ }^{1}$ and $\mathrm{Xu}$ et al. (2005), respectively, and they have been re-analyzed here using the same method applied to the other groups. Tzanavaris et al. (2009) used SWIFT/UVOT to analyze the UV properties of another sample of Hickson Compact Groups of galaxies which will be complementary to our GALEX data.

\subsection{R-band data}

$R$-band images of HCG 2, 23 and 100 were obtained at the Cerro Tololo Interamerican Observatory on Aug. 23/2005, using the $4 \mathrm{~m}$ Blanco telescope and a mosaic II CCD imager, with a pixel scale of $0.27 \mathrm{arcsec} / \mathrm{pixel}$, for a field of view of $40 \times 40 \mathrm{arcmin}$, centered on each group. For each target, three exposures of $300 \mathrm{~s}$ were taken in the $R$ band, with a typical seeing of $1.1 \mathrm{arcsec}$. The data were bias subtracted and flat-fielded using IRAF $^{2}$, using standard procedures (package mscred). A flat-field was constructed from a combination of dark sky frames and twilight flats which worked well, given that the background, after flat-fielding, showed an rms variation lower than $1 \%$ over the whole field. Since the night was not photometric, no calibration stars were observed and the zeropoints were obtained from published surface brightness profiles of group members (Mendes de Oliveira 1992; Rubin et al. 1991).

HCG 22 was observed with the Keck II telescope in the $R$ and $B$ bands. These data are described in da Rocha et al. (2002).

The $R$-band image of NGC 92 was obtained at La Silla Observatory with the NTT telescope. It was taken on Oct. 27th 1994, within a program to monitor the supernova SN1994Z, which was discovered in October 1994, in the galaxy NGC 87, a close companion to NGC 92. The seeing was $1^{\prime \prime}$ and the pixel scale was 0.27 "/pixel. The zeropoint was estimated with calibration stars taken on the same night as the targets.

\footnotetext{
1 de Mello et al. (2008a) selected the UV sources based on proximity to the HI tail while in this work, sources were selected within a region of radius equal to two times the radius of the minimum circle, as defined in Sect. 3.2.

2 IRAF is distributed by the National Optical Astronomy Observatories, which are operated by the Association of Universities for Research in Astronomy, Inc., under cooperative agreement with the National Science Foundation.
} 
Table 1. Properties of the sample.

\begin{tabular}{|c|c|c|c|c|c|c|c|c|c|c|}
\hline \multirow[b]{2}{*}{ name } & \multirow[b]{2}{*}{$\begin{array}{c}\text { Morph. }^{a} \\
\text { type }\end{array}$} & \multirow[b]{2}{*}{$\begin{array}{c}\text { distance }^{b} \\
\text { Mpc }\end{array}$} & \multirow[b]{2}{*}{$\begin{array}{l}B_{\mathrm{TC}}{ }^{a} \\
\mathrm{mag}\end{array}$} & \multirow[b]{2}{*}{$\begin{array}{c}V_{\text {sys }}{ }^{a} \\
\mathrm{~km} \mathrm{~s}^{-1}\end{array}$} & \multirow[b]{2}{*}{$\begin{array}{c}V_{\text {sys }}{ }^{c} \\
\mathrm{~km} \mathrm{~s}^{-1}\end{array}$} & \multicolumn{3}{|c|}{ Position angle (PA (deg)) } & \multicolumn{2}{|c|}{ Inclination (deg) } \\
\hline & & & & & & $\begin{array}{c}\text { velocity } \\
\text { Map }\end{array}$ & $\begin{array}{c}\text { optical }^{d} \\
\text { image }\end{array}$ & $\begin{array}{c}\text { optical }^{e} \\
\text { image }^{2}\end{array}$ & $\begin{array}{c}\text { velocity } \\
\text { Map }\end{array}$ & $\begin{array}{l}\text { optical } \\
\text { image }\end{array}$ \\
\hline \multicolumn{11}{|l|}{ HCG 2} \\
\hline $\mathrm{a}$ & SBd & 62.36 & 13.35 & 4326 & 4347 & $41 \pm 3$ & 3 & 8 & $68 \pm 13$ & 61 \\
\hline $\mathrm{b}$ & $\mathrm{cI}$ & 62.36 & 14.39 & 4366 & 4357 & $8 \pm 3$ & 28 & 3 & $49 \pm 12$ & 30 \\
\hline $\mathrm{c}$ & $\mathrm{SBc}$ & 62.36 & 14.15 & 4235 & 4266 & $168 \pm 3$ & 132 & 158 & $\ldots$ & 57 \\
\hline \multicolumn{11}{|l|}{ HCG 7} \\
\hline $\mathrm{a}$ & $\mathrm{Sb}$ & 61.04 & 12.98 & 4210 & 4198 & $176 \pm 1$ & 153 & 165 & $67 \pm 3$ & 61 \\
\hline $\mathrm{b}$ & SB0 & 61.04 & 13.74 & 4238 & & & & & & \\
\hline $\mathrm{c}^{f}$ & $\mathrm{SBc}$ & 61.04 & 12.60 & 4366 & & 133 & 133 & 144 & 48 & 36 \\
\hline $\mathrm{d}$ & $\mathrm{SBc}$ & 61.04 & 14.77 & 4116 & 4104 & $85 \pm 3$ & 58 & 35 & $31 \pm 12$ & 43 \\
\hline \multicolumn{11}{|l|}{ HCG 22} \\
\hline $\mathrm{a}$ & E2 & 38.81 & 12.24 & 2705 & & & & & & \\
\hline b & $\mathrm{Sa}$ & 38.81 & 14.47 & 2625 & & & & & & \\
\hline $\mathrm{c}$ & SBcd & 38.81 & 13.90 & 2728 & 2572 & $101 \pm 2$ & 70 & 179 & $\ldots$ & 25 \\
\hline \multicolumn{11}{|l|}{ HCG 23} \\
\hline $\mathrm{a}$ & $\mathrm{Sab}$ & 69.81 & 14.32 & 4798 & & & & & & \\
\hline b & $\mathrm{SBc}$ & 69.81 & 14.42 & 4921 & & & & & & \\
\hline $\mathrm{c}$ & So & 69.81 & 15.52 & 5016 & & & & & & \\
\hline d & $\mathrm{Sd}$ & 69.81 & 16.00 & 4562 & & & & & & \\
\hline \multicolumn{11}{|l|}{ HCG 92} \\
\hline $\mathrm{b}$ & Sbc & 93.60 & 13.18 & 5774 & & & & & & \\
\hline $\mathrm{c}$ & $\mathrm{SBc}$ & 93.60 & 13.33 & 6764 & & & & & & \\
\hline d & $\mathrm{Sc}$ & 93.60 & 13.63 & 6630 & & & & & & \\
\hline $\mathrm{e}$ & E1 & 93.60 & 14.01 & 6599 & & & & & & \\
\hline \multicolumn{11}{|c|}{ HCG $100^{g}$} \\
\hline $\mathrm{a}$ & $\mathrm{Sb}$ & 77.28 & 13.66 & 5300 & 5323 & $78 \pm 5$ & 78 & 79 & $50 \pm 7$ & 47 \\
\hline $\mathrm{b}$ & $\mathrm{Sm}$ & 77.28 & 14.90 & 5253 & 5163 & $145 \pm 5$ & 165 & 149 & $52 \pm 10$ & 57 \\
\hline $\mathrm{c}$ & $\mathrm{SBc}$ & 77.28 & 15.22 & 5461 & 5418 & $72 \pm 8$ & 70 & 67 & $66 \pm 5$ & 65 \\
\hline d & Scd & $\ldots$ & 15.97 & $\ldots$ & & $53 \pm 3$ & 47 & 50 & $70 \pm 5$ & 64 \\
\hline \multicolumn{11}{|l|}{ NGC 92} \\
\hline NGC 92 & $\mathrm{Sa}$ & 48.50 & $\ldots$ & 3219 & 3412 & $148 \pm 3$ & 149 & 149 & $48 \pm 6$ & 62 \\
\hline NGC 89 & $\mathrm{SB}(\mathrm{s}) 0 / \mathrm{a}$ & 48.50 & $\ldots$ & 3320 & & & & & & \\
\hline NGC 88 & $\mathrm{SB}(\mathrm{rs}) 0 / \mathrm{a}$ & 48.50 & $\ldots$ & 3433 & & & & & & \\
\hline NGC 87 & $\mathrm{IBm}$ & 48.50 & $\ldots$ & 3491 & & & & & & \\
\hline
\end{tabular}

${ }^{a}$ Taken from Hickson (1993) and from NED database for NGC 92. Morphological type for NGC 92 was taken from Prugniel et al. (1998).

${ }^{b}$ Distances were obtained using the redshift for each group given in Hickson (1992) and assuming the groups follow the Hubble flow. For NGC 92, we used the mean redshift of the group members. For each galaxy, redshift was taken from NED. In all cases, we used $H_{0}=70 \mathrm{~km} \mathrm{~s}^{-1} \mathrm{Mpc}^{-1}$, $\Omega_{\mathrm{m}}=0.3$ and $\Omega_{\lambda}=0.7$.

${ }^{c}$ Systemic velocity deduced from our velocity field analysis.

${ }^{d}$ For HCG 2a, b, c, HCG 7a, d, HCG 22c, and values of PA were taken from Mendes de Oliveira (1992). These were determined at semimajor axis lengths of $2 h^{-1} \mathrm{kpc}$ for all Hickson group galaxies with $z \leq 0.05$. For NGC 92 the PA was taken from the Hyperleda database.

${ }^{e}$ Taken from the Hyperleda database. PA is the position angle of the major axis at the $25 \mathrm{mag} \operatorname{arcsec}^{-2}$ isophote, in the $B$-band.

$f$ Taken from Mendes de Oliveira et al. (2003).

${ }^{g}$ Taken from Plana et al. (2003).

HCG 92 was observed with Gemini in the Sloan filters $g$, $r$ and $i$. These data are described in Mendes de Oliveira et al. (2004). For HCG 7, the $r$-band image of the Sloan Digital Sky Survey (SDSS) was used in our analysis. Transformation between $r$-band magnitudes and $R$-band magnitudes was performed using $R=r-0.1837 \times(g-r)$ (Lupton 2005) for the sources in groups HCG 7 and 92.

\section{Data analysis}

\subsection{Rotation curves and kinematic parameters}

In order to constrain the evolutionary stage of each compact group, we inspected the velocity field and rotation curve of each member to search for interaction indicators ${ }^{3}$. Rotation curves were constructed using the morphological center (defined as the location of the peak intensity in the continuum image) instead of the kinematic center (defined as the center that produces a symmetric rotation curve) because in most cases the rotation curves were asymmetric. Asymmetry made it difficult to match both sides of the curve, no matter which center was chosen. In most cases there was clearly a decoupling between the morphological and kinematic centers (see Amram et al. 2007). Kinematic position angles, inclinations, systemic velocities (see Table 1) and

\footnotetext{
3 Early-type galaxies HCG 7b and HCG 22b (Table 1) had very weak $\mathrm{H} \alpha$ emission and, therefore, no rotation curves were derived for these objects.
} 

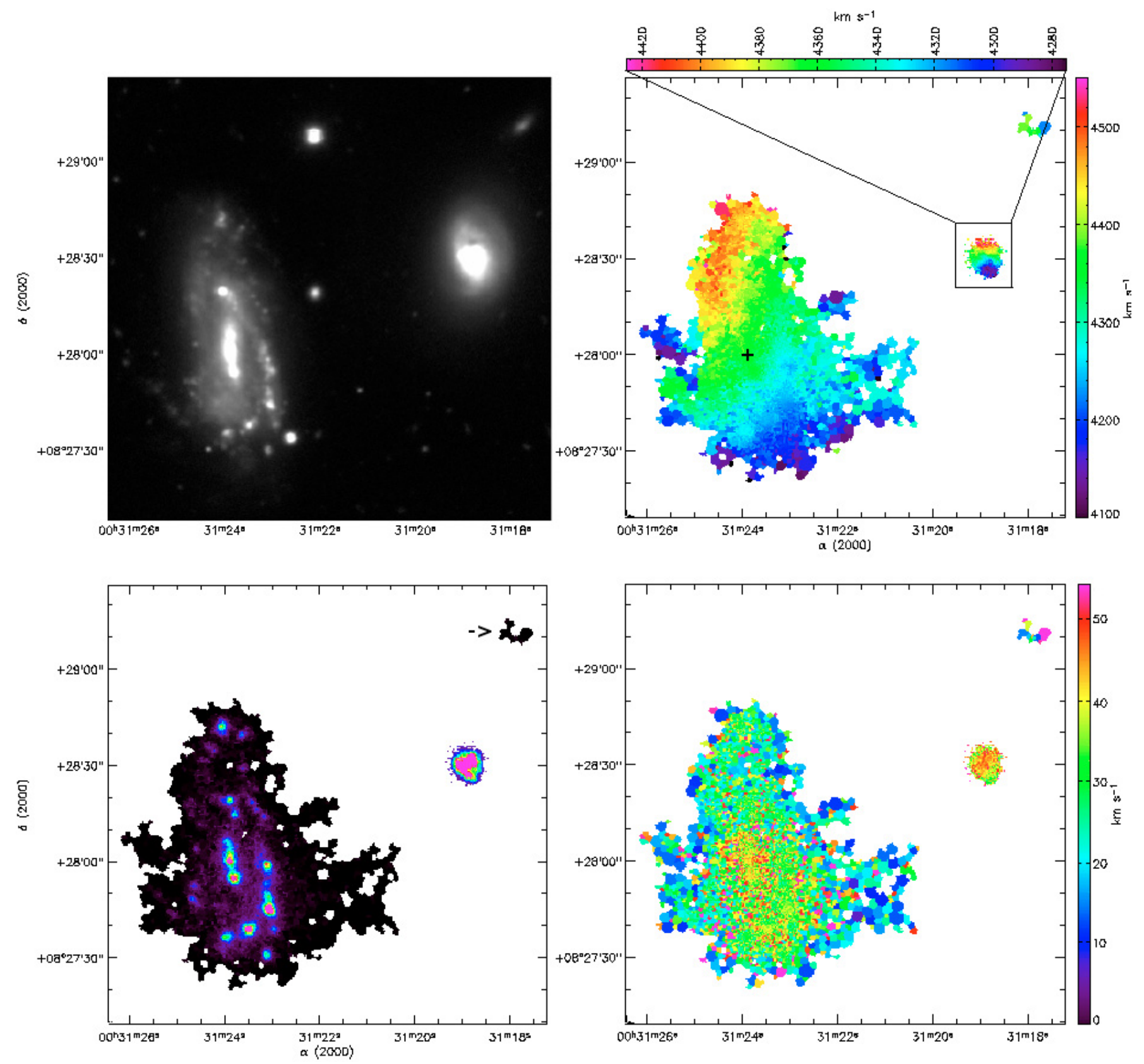

Fig. 1. HCG 2a,b. North is at the top and East is to the left of all images in Figs. 1 to 6. HCG 2a is located to the East of HCG 2b. Top left: $R$ band image from CTIO. Top right: velocity field. We highlight the HCG $2 \mathrm{~b}$ scale at the top of the velocity field, in order to show clearly its dynamic range. Bottom left: monochromatic image. Bottom right: velocity dispersion map. Small arrow in the $R$ band image and monochromatic map indicates the position of a new source (probably in the background) detected in the Fabry-Perot analysis.

rotation curves were derived from the velocity fields using the procedure developed by Epinat et al. (2008a). The position of the morphological center for each galaxy is marked with a plus sign in the velocity fields of Figs. 1 to 6.

The optical position angles (PAs) were taken from Mendes de Oliveira (1992) and from the Hyperleda ${ }^{4}$ database.

In all cases, the PA is oriented from North to East. For HCG 2c and HCG 22c, the kinematic inclination resulted in unrealistic values which resulted in maximum rotation velocities higher than the expected velocities (Torres-Flores et al. 2009). Therefore, in these cases, we used the morphological inclinations obtained from the relation $\cos (b / a)=i$, where $b$ and $a$

\footnotetext{
$\overline{{ }^{4} \text { http://leda.univ-lyon } 1 . f r}$
}

correspond to the length of the minor and major axis of the $\mu_{B}=$ 25 mag arc ${ }^{-2}$ isophote given in Hickson (1993).

\subsection{Source extraction and photometry}

We searched for ultraviolet emitting regions in the vicinity of all seven targets (see Sect. 4.2 for the corresponding figures). We also include HCG 92 and HCG 100 which were previously analyzed in Mendes de Oliveira et al. (2004) and de Mello et al. (2008a), using the following method. We generated catalogs using SExtractor (SE, Bertin \& Arnouts 1996) in the FUV, NUV and $R$-band sky-subtracted images. The threshold for source detection was set to 1.5 sigma $($ DETECT_THRESH $=1.5$ ) for a minimum area of 5 pixels $($ DETECT_MINAREA $=5$ ). 

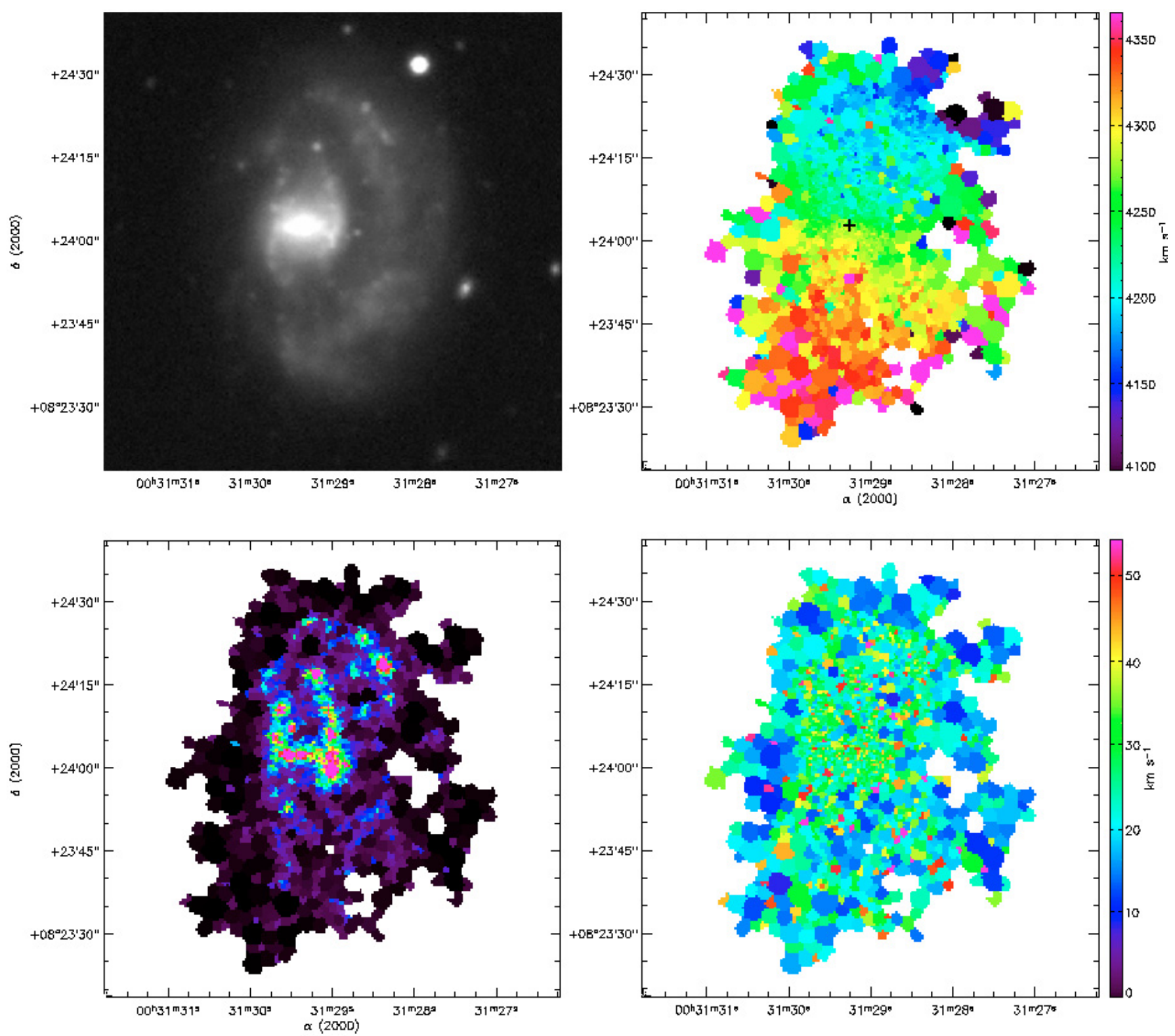

Fig. 2. HCG 2c. Top left: $R$ band image from CTIO. Top right: velocity field. Bottom left: monochromatic image. Bottom right: velocity dispersion map.

We recorded, for each source, the right ascention, declination, MAG_AUTO (AB system) and Petrosian radius. We used SE Kron elliptical apertures to measure total magnitudes (MAG_AUTO) in FUV, NUV and $R$-bands. Although MAG_AUTO is often used to measure total magnitudes in galaxy surveys (e.g. Bell et al. 2004; de Mello et al. 2006; Zucca et al. 2006), high uncertainties might be expected at the faint magnitudes due to the assumption that the sky background has Gaussian random noise without source confusion (Brown et al. 2007). However, since we are comparing data taken with different resolutions and the fact that UV light does not necessarily peak at the same coordinate as the optical light, MAG_AUTO performs better than the other SExtractor parameter choices, as long as a careful match between the different catalogs is performed (see de Mello et al. 2008a). We first matched the FUV and NUV catalogs using a $3^{\prime \prime}$ radius and as a result we obtained the FUV and NUV parameters for each detected source. We then matched the UV catalog with the $R$-band catalog. Therefore, only objects with FUV detections were included in our final catalog. Due to the large GALEX field of view (1.28 and 1.24 in FUV and NUV, respectively), we performed the source detection in four regions for each GALEX image: one field centered on the centroid of the group (on-group) and three other fields (each with $\sim 15^{\prime} \times 15^{\prime}$ ) distributed over the outskirts of the images (hereafter, control sample, CS). The on-group field was defined within a region of radius equal to two times the radius of the minimum circle that passes through the center of each group member. This area was chosen because it usually encompasses the group and its HI debris, if there is any.

Given that we used a different exposure time for each compact group observation, we had to limit our analysis to the brightest completeness limit, which was that for the field of HCG 92, at an NUV magnitude of 22.2. The colors FUV-NUV and FUV- $R$ were estimated inside a fixed aperture of 4 " radius, centered on the centroid of the light distribution of each NUV band detection, using the task PHOT in IRAF. Total magnitudes and fixed 

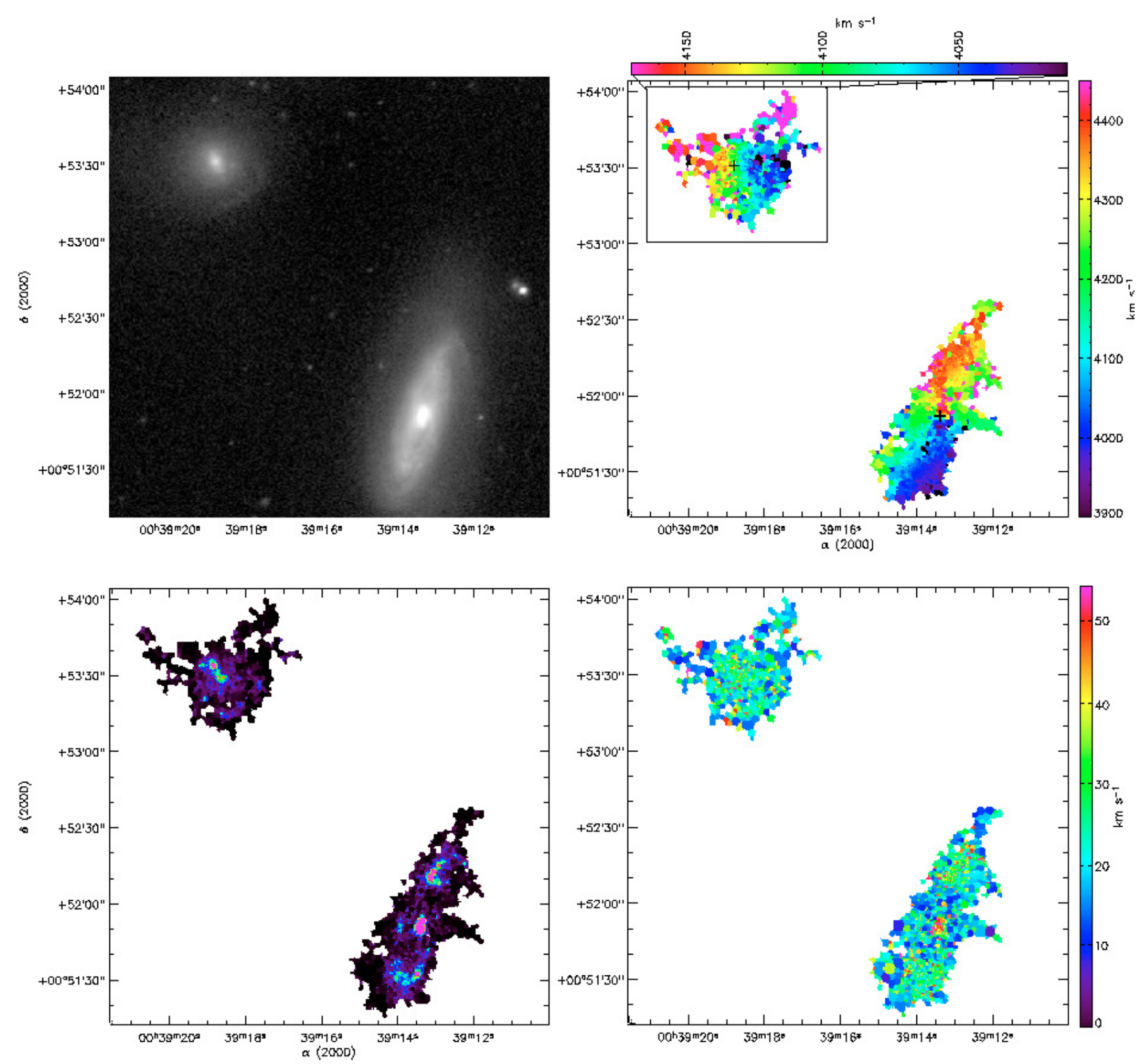

Fig. 3. HCG 7a,d. HCG 7a is located Southwest of HCG 7d. Top left: $r$ band image from SDSS. Top right: velocity field. We highlight the HCG 7d scale at the top of the velocity field, in order to show clearly its dynamic range. Bottom left: monochromatic image. Bottom right: velocity dispersion map.

aperture magnitudes were corrected for galactic extinction, using $A_{\mathrm{FUV}}=E(B-V) \times 8.29, A_{\mathrm{NUV}}=E(B-V) \times 8.18$ (Seibert et al. 2005) and $A_{R}=E(B-V) \times 2.634$ (Schlegel et al. 1998), following de Mello et al. (2008a).

\section{Results}

\subsection{Kinematic properties of the main galaxies}

In this section, we analyze each individual galaxy based on our optical $R$-band images, velocity fields, monochromatic images, velocity dispersion maps (Figs. 1 to 6), and rotation curves (Figs. 7 and 8), together with information from the literature. Note that the dispersion velocity maps were corrected for instrumental broadening, measured using the calibration cube. We estimated that the broadening is, on average, $24.8 \mathrm{~km} \mathrm{~s}^{-1}$ accross the field. In the last columns of Table 1, we list the kinematic parameters obtained from the velocity fields. In Cols. 5 and 6 of Table 1 the systemic velocities taken from the literature and the velocities resulting from our analysis are listed.

\subsubsection{HCG 2}

This compact group is formed of three galaxies with corresponding redshifts. According to the NED ${ }^{5}$ database and the RC3 catalogue (de Vaucouleurs et al. 1991), the brightest galaxy of the system, HCG 2a, is classified as a late-type barred SBd galaxy. HCG $2 b$ is classified as a cI (compact Irr) by Hickson (1993) and it is the strongest FIR source in this group (Allam et al. 1996).

$\overline{5 \text { http://nedwww.ipac.caltech.edu/ }}$ 

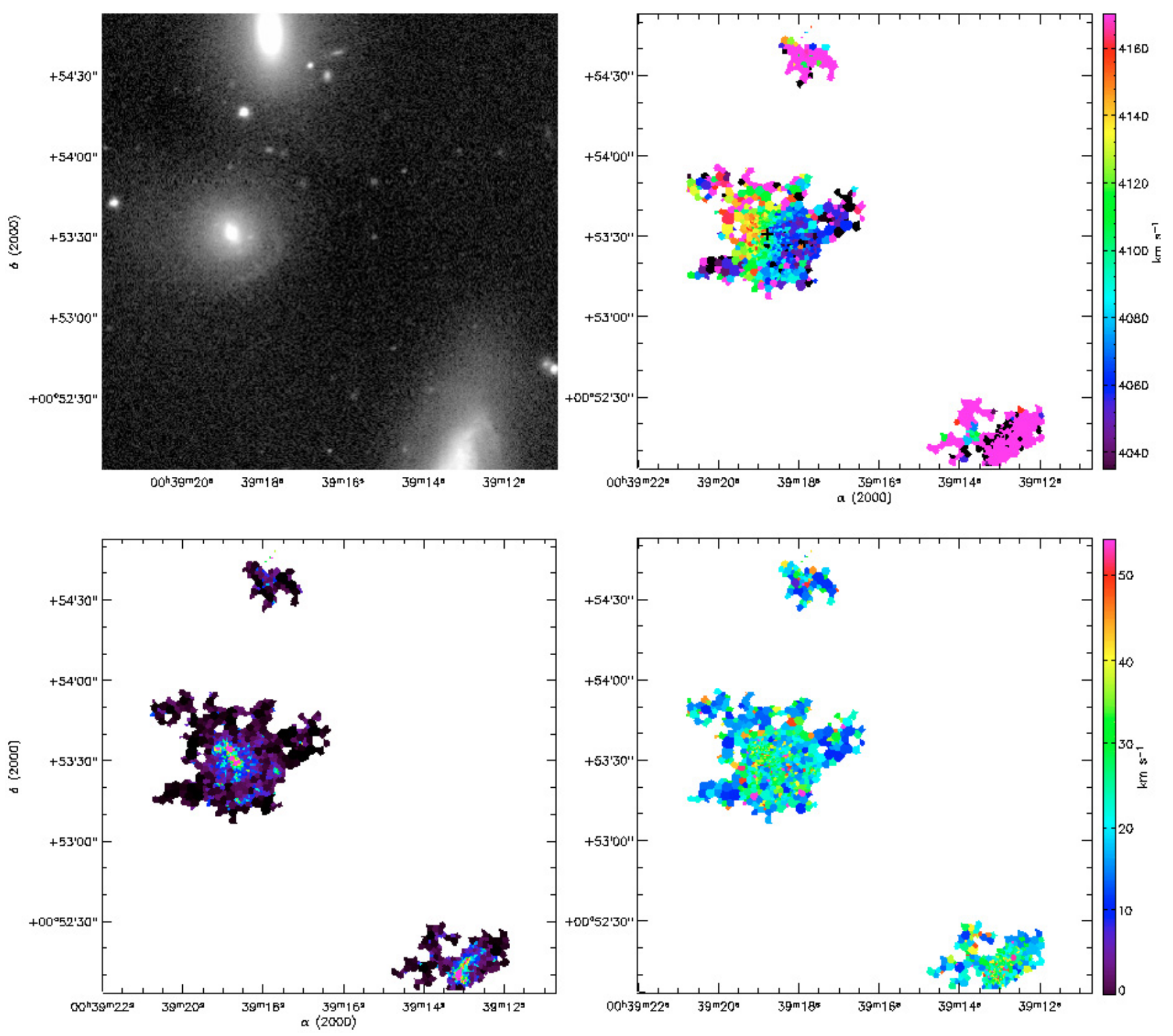

Fig. 4. HCG 7b,d. HCG 7d is located in the center of the field. HCG 7a and b are partially shown in the South and North of the field respectively. Top left: $r$ band image from SDSS. Top right: velocity field. Bottom left: monochromatic image. Bottom right: velocity dispersion map.

HCG 2c is an SBc-type galaxy. Figure 1 shows the velocity field, the velocity dispersion, monochromatic and continuum maps for HCG 2a,b.

HCG 2a shows a very disturbed velocity field, as can be seen in Fig. 1. No symmetry is observed with respect to the major axis and moreover one cannot see a constant velocity along the minor axis. The PA of the velocity field changes rapidly, by about $90^{\circ}$ from the North to the South of the galaxy. The velocity field shows a clear signature of a strong bar, almost vertical, which is responsible for the PA variation and for the misalignment between the morphological and kinematic orientations. There is a misalignment of $30^{\circ}$ between the bar and the kinematic minor axis. This type of velocity field has been observed in Epinat et al. (2008b) (e.g. UGC 12754, UGC 11407 and UGC 1256). Given that the kinematic PA is so perturbed by the bar, it is better to use the morphological PA to draw the RC (see Epinat et al. 2008a). The monochromatic map shows several HII regions. In the southern side, the regions are aligned along the spiral arm. Intense HII regions in the center of the object belong to the northern arm or to the bar. Towards the northern side of the galaxy only one bright region is visible, although other fainter HII regions are also seen around the same location. On the south side, we detected an extension of $\mathrm{H} \alpha$ emission towards the West of this object. This signature was not reported in Vilchez et al. (1998). The dispersion map shows higher velocity dispersion in areas where HII regions are present (Fig. 1). The velocity dispersion within HII regions along the bar is $41 \mathrm{~km} \mathrm{~s}^{-1}$ while outside these regions the dispersion is $25 \mathrm{~km} \mathrm{~s}^{-1}$. The velocity dispersion increases along the spiral arm, from $26 \mathrm{~km} \mathrm{~s}^{-1}$ to $40 \mathrm{~km} \mathrm{~s}^{-1}$.

The rotation curve of HCG $2 \mathrm{a}$ shown in Fig. $7 \mathrm{a}$ is highly asymmetric, with the receding side reaching $326 \mathrm{~km} \mathrm{~s}^{-1}$ and the approaching side $270 \mathrm{~km} \mathrm{~s}^{-1}$, for a systemic velocity of $4347 \mathrm{~km} \mathrm{~s}^{-1}$. The approaching side slope of the rotation curve is steeper than the receding side on the SW side. For the adopted systemic velocity ( $4347 \mathrm{~km} \mathrm{~s}^{-1}$ ), the rotation curve does not pass through the center of the diagram. However, both sides of the 

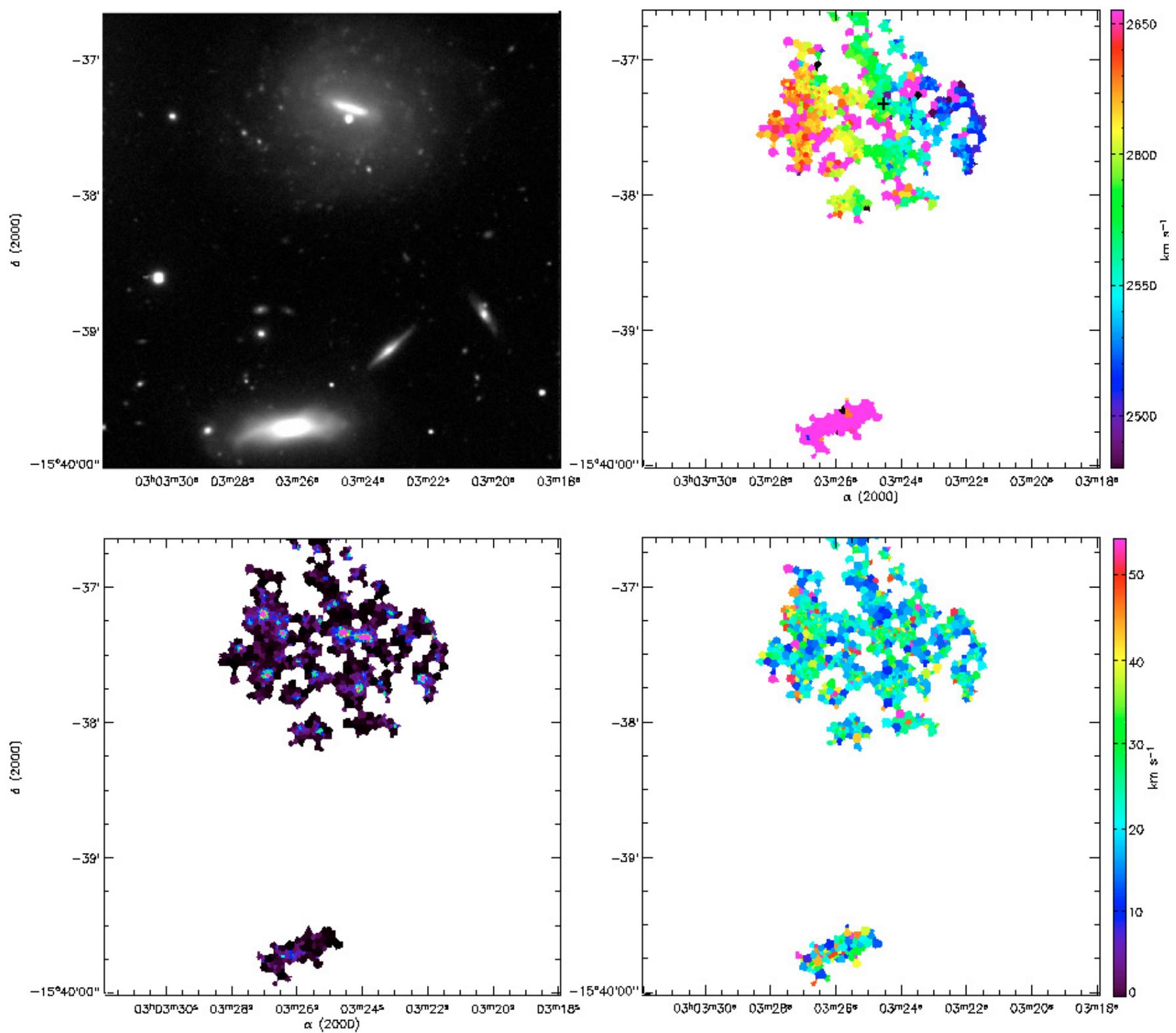

Fig. 5. HCG 22b,c. HCG 22b lies South of HCG 22c. Top left: $R$ band image from CTIO. Top right: velocity field. Bottom left: monochromatic image. Bottom right: velocity dispersion map.

rotation curve match in the external region of the galaxy (outside $\left.10^{\prime \prime}\right)$. The center of the curve is particularly disturbed due to the strong bar. In this region, the rotation curve is flat with a few oscillations within 10 arcsec on both sides. The flatness of the curve is due to the orientation of the bar with respect to the major axis of the galaxy (Hernandez et al. ?, in preparation). The center of the bar coincides with the center of the external ellipses of the continuum (for radii outside $r=12^{\prime \prime}$ in the minor axis and $r=22^{\prime \prime}$ in the major axis).

In the case of HCG $2 \mathrm{~b}$, we detected $\mathrm{H} \alpha$ emission at its center (within $2.8 \mathrm{kpc}$, also reported in Vilchez et al. 1998). The velocity field (Fig. 1) seems to be regular. The kinematic position angle differs from the morphological one (Mendes de Oliveira et al. 1992) by $20^{\circ}$ at $2 h^{-1} \mathrm{kpc}$, however, at $R_{25}$, the optical position angle is in good agreement with the kinematic one. We noticed that the continuum and monochromatic images peak at the same position. The galaxy center displays a velocity dispersion of $47 \mathrm{~km} \mathrm{~s}^{-1}$ while in the outskirts the value is $34 \mathrm{~km} \mathrm{~s}^{-1}$.
We can also notice that a high value for the dispersion often corresponds to strong monochromatic emission. The rotation curve of HCG 2b (Fig. 7b) shows a disk-like rotator shape. For the adopted systemic velocity, both sides match at radii larger than $2^{\prime \prime}$, however, in the inner part of the rotation curve (inside $2^{\prime \prime}$ ) the approaching side shows a plateau and the two sides do not match. We detected two bumps in the approaching side of the rotation curve, placed between $0^{\prime \prime}$ and $4^{\prime \prime}$ and $4^{\prime \prime}$ and $7 "$, respectively.

The velocity field of HCG $2 \mathrm{c}$ is regular (see Fig. 2). A signature of the bar is suspected in the central regions. The PA is almost constant along the major axis, staying around a value of $168^{\circ}$. The monochromatic map shows several bright HII regions in the bar, in the center of the galaxy and across the northern arm. A tidal arm is identified in the monochromatic map, in a similar way to UGC 7861, as was reported by Epinat et al. (2008a). There are no bright HII regions on the south side of this object. The velocity dispersion map does not reveal any 

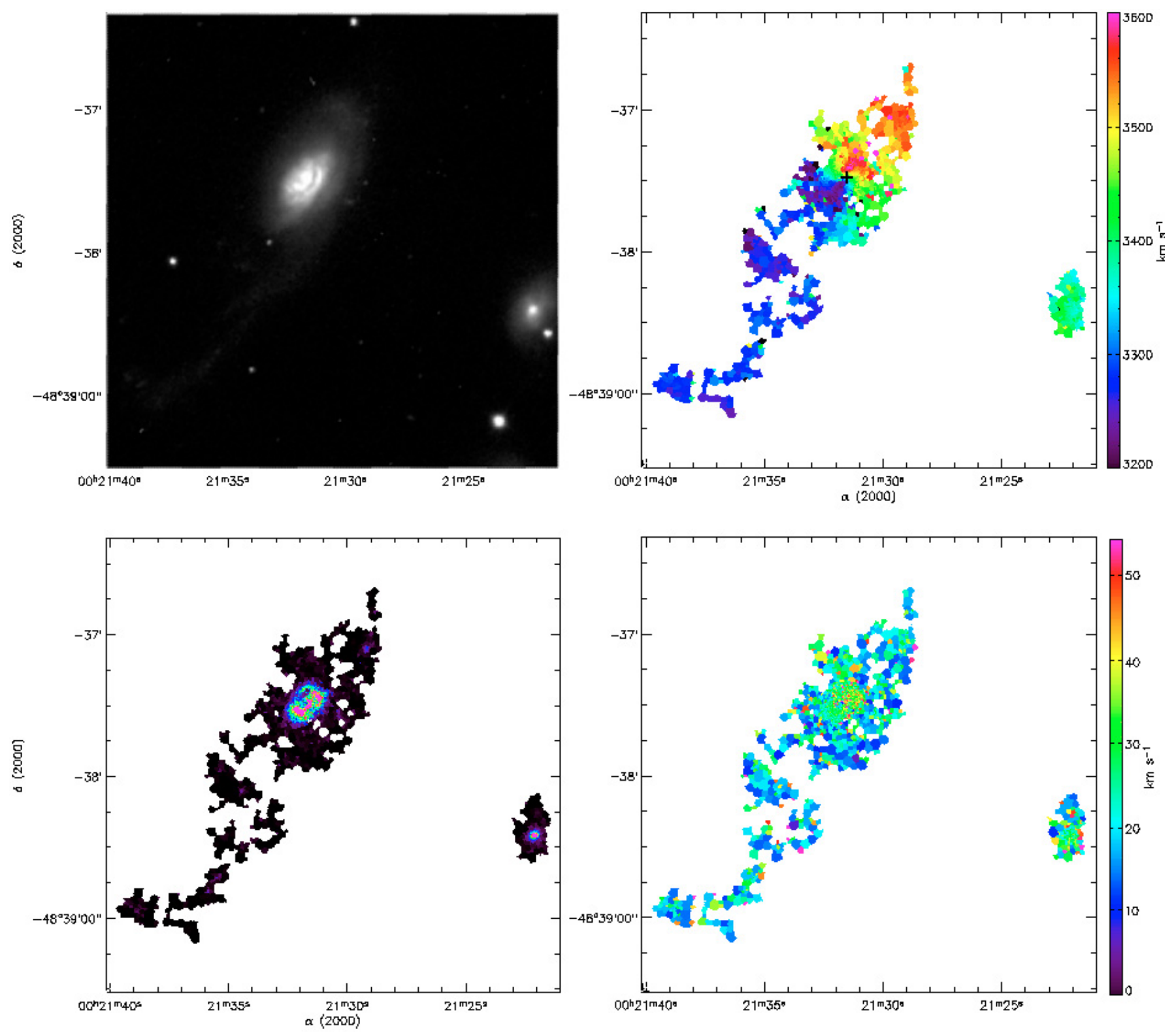

Fig. 6. NGC 92 and NGC 88. NGC 92 lies East of NGC 88. Top left: $R$ band image from NTT. Top right: velocity field. Bottom left: monochromatic image. Bottom right: velocity dispersion map.

peculiarities. HCG 2c displays a more symmetric rotation curve than the other two members of this group, except in the central regions $\left(\leq 10^{\prime \prime}\right)$. The velocity amplitude is $\sim 110 \mathrm{~km} \mathrm{~s}^{-1}$, across $30^{\prime \prime}(8 \mathrm{kpc})$. We also notice that the continuum center is $2^{\prime \prime}(0.5 \mathrm{kpc})$ away from the kinematic center, towards the SE of the galaxy.

The emission detected in the upper right corner of Fig. 1 seems to be from a background galaxy, i.e., an emission line falling in the same velocity range as $\mathrm{H} \alpha$ for HCG 2. Its morphology, as per inspection of the CTIO image of the group, seems to be that of a giant galaxy at a high redshift.

\subsubsection{HCG 7}

This group is formed by four objects, three late-type galaxies $(\mathrm{Sb}, \mathrm{SBc}$ and $\mathrm{SBc}$ ) and one early-type (SB0) galaxy (Hickson 1993). HCG 7a and HCG 7c are strong FIR sources
(Allam et al. 1996; Verdes-Montenegro et al. 1998). Shimada et al. (2000) classified the nuclear activity in HCG 7a as an HII type.

HCG 7a shows $\mathrm{H} \alpha$ emission in several knots through the arms (see Fig. 3) as was also noted in Vilchez et al. (1998). The central region displays a peak in the monochromatic and continuum image. The velocity field of this galaxy is very peculiar, strong asymmetries around the major axis are observed on both sides of the galaxy. Moreover, in the center (inside $3 \mathrm{kpc}$ ), strong non-circular motions are observed with the velocities reaching high values of $290 \mathrm{~km} \mathrm{~s}^{-1}$ in the rotation curve (see Fig. 7d). The velocity dispersion map for HCG 7a displays a high value of $55 \mathrm{~km} \mathrm{~s}^{-1}$ in the center of the galaxy, corresponding to the central HII region visible in the monochromatic map. The other HII regions of this galaxy do not show high velocity dispersions. The bump in the central region of the rotation curve has already been found in other galaxies such as UGC 6118 

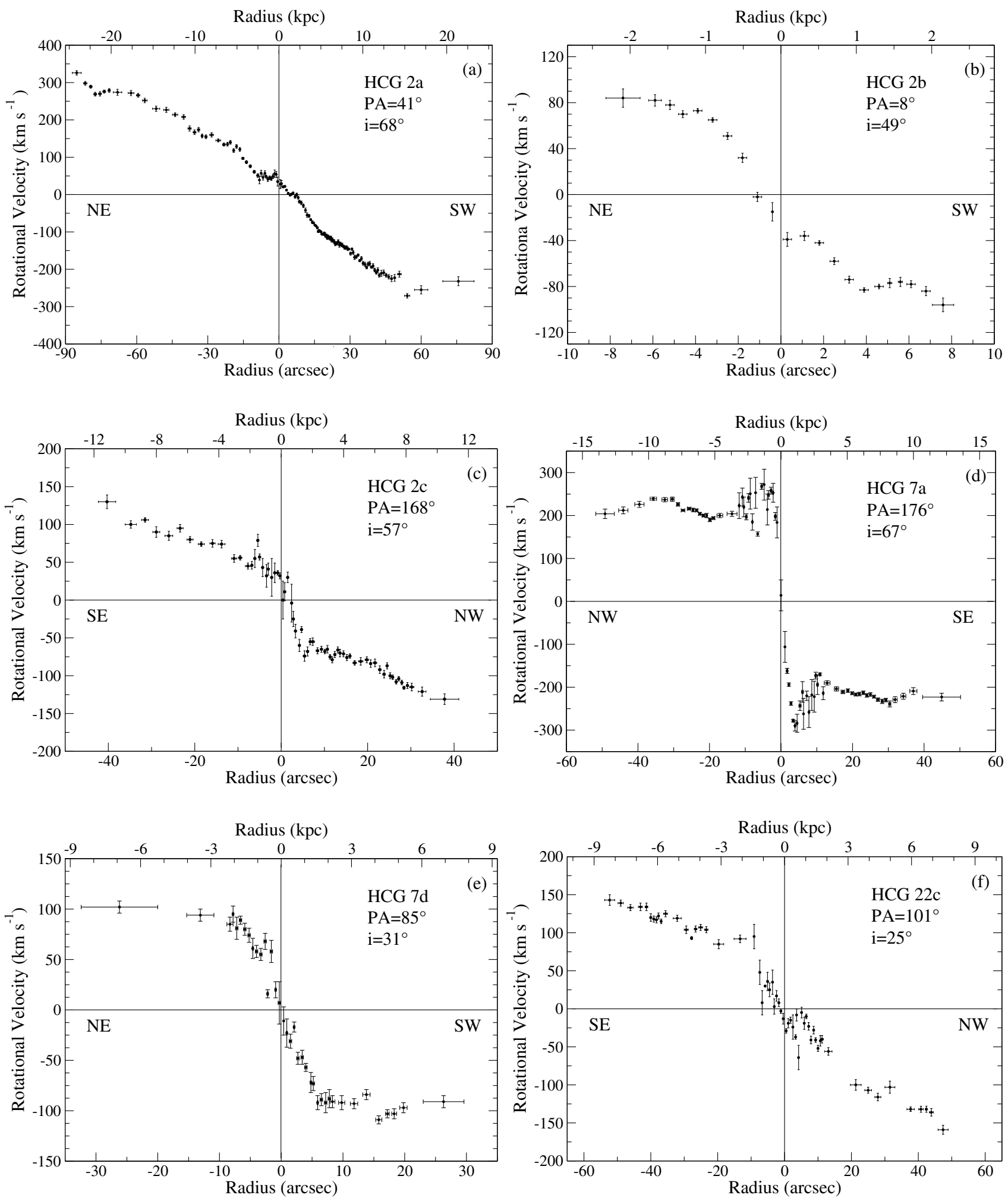

Fig. 7. Rotation curves for individual galaxies in our sample. The parameters used to derive the rotation curves are given in each plot (PA: position angle, $i$ : inclination). The adopted galaxy center is the morphological center. The horizontal error bars represent the $\pm 1 \sigma$ radius dispersion weighted by $\cos (\theta)$, where $\theta$ is a polar coordinate in the plane of the galaxy.

(Epinat et al. 2008a). The peak in the inner part corresponds to a region of high monochromatic emission and might be the result of a small bar, associated with the HII regions in the central parts. However, there is no previous mention of a stellar bar present for this galaxy. Both stellar and gaseous major axes appear to be aligned, probably due to the high inclination of the galaxy.
The SBc galaxy HCG 7d has been observed twice; the two observations are presented in Figs. 3 and 4. This galaxy shows strong $\mathrm{H} \alpha$ emission at the end of its bar, visible in the HST archival image and in several knots in their southwest arm. On the northeast side of this object, the intensity of $\mathrm{H} \alpha$ emission is lower than that on the south-west side. The monochromatic 


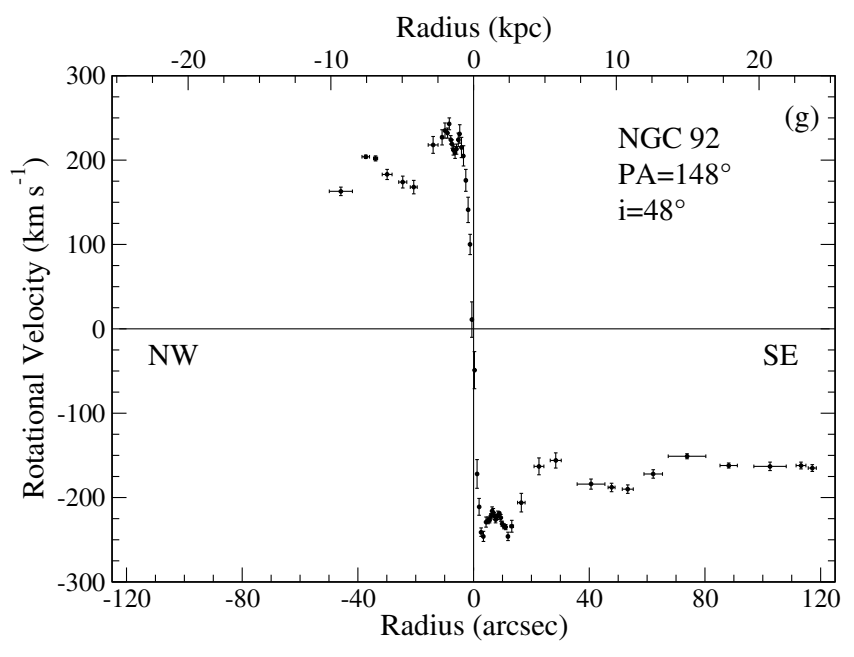

Fig. 8. Rotation curve for NGC 92. See also caption of Fig. 7.

map of HCG 7d shown in Fig. 4 displays emission in the east and west side of the galaxy, which is not detected in the monochromatic image of Fig. 3 or in the $r$-band image. We suspect that this signature is noise or residual emission from the $\mathrm{OH}$ subtraction. The velocity field of HCG $7 \mathrm{~d}$ clearly shows the presence of the bar (see Fig. 4). The kinematic PA differs by $27^{\circ}$ and $50^{\circ}$ compared to the optical PA given in Mendes de Oliveira et al. (2003) and Hyperleda, respectively. The velocity dispersion map does not show any particular features. The rotation curve is quite regular, reaching a plateau of $100 \mathrm{~km} \mathrm{~s}^{-1}$ from $7(2 \mathrm{kpc})$ to $30^{\prime \prime}$ $(8 \mathrm{kpc})$. We conclude that this galaxy has a regular velocity field and rotation curve.

HCG 7b shows a weak $\mathrm{H} \alpha$ emission in its center but the observations are truncated by the edge of the field of view. No information can be extracted from this velocity field.

Mendes de Oliveira et al. (2003) analyzed HCG 7c using Fabry-Perot data and did not find peculiarities in its velocity field.

We note that the rotation curves for the galaxies in this group do not reach the optical radius $R_{25}$, except for HCG 7c. This could be related to the fact that in HCG 7 about $80 \%$ of the HI content of the member galaxies is missing (Verdes-Montenegro et al. 2001).

\subsubsection{HCG 22}

This group, composed of a triplet of galaxies with concordant redshifts, contains a bright elliptical and two spiral galaxies in its core (morphological types E2, Sa and SBcd according to Hickson 1993). Coziol et al. (1998) classified the nuclear region of HCG 22a as a dwarf Seyfert 2 and Gallagher et al. (2008) did not find evidence for any excess mid-infrared emission in this object. Verdes-Montenegro et al. (1998) gave upper limits for the IR luminosities of HCG 22b and HCG 22c $\left(\log L_{\mathrm{IR}}<\right.$ $8.55 L_{\odot}$ and $\log L_{\mathrm{IR}}=8.88 L_{\odot}$ respectively).

The monochromatic map of HCG 22c (in Fig. 5) shows several HII regions that belong to the arms of this galaxy, with a patchy appearance. Moreover, there is faint $\mathrm{H} \alpha$ emission in the intra-arm region. The velocity field seems to be regular, with a misalignment of about $30^{\circ}$ between the kinematic and optical PA. The velocity dispersion map does not show any particular features. The rotation curve displayed in Fig. $7 \mathrm{f}$ shows almost a linear shape, like a solid-body rotator, particularly in the northwest part of the galaxy, steadily rising from 0 to $125 \mathrm{~km} \mathrm{~s}^{-1}$ out to $30^{\prime \prime}(5 \mathrm{kpc})$. From $30^{\prime \prime}(5 \mathrm{kpc})$ to $45^{\prime \prime}(7 \mathrm{kpc})$ the velocity rises from $125 \mathrm{~km} \mathrm{~s}^{-1}$ to $170 \mathrm{~km} \mathrm{~s}^{-1}$. In the inner part of the rotation curve (inside $15^{\prime \prime}$ ), the approaching and receding sides do not match; however, from 20 to 45", both sides are in agreement.

The early-type spiral HCG 22b only shows weak $\mathrm{H} \alpha$ emission in its central region. Therefore, the velocity field, which appears to be uniform, is poorly determined and it is not possible to obtain a rotation curve with the data in hand. HCG22b shows a high velocity dispersion equal to $48 \mathrm{~km} \mathrm{~s}^{-1}$ in its center, which corresponds to the bright emission visible in the monochromatic map. HCG 22b exhibits extended shells clearly seen in the $B$ and $R$-band images, as shown in Fig. 9.

\subsubsection{NGC 92}

This galaxy is part of the Phoenix compact group formed by NGC 92, NGC 88 and NGC 89 and NGC 87 (Rose 1979). This group is also known as Rose 34 (Rose 1977) and SCG 00184854 (Iovino et al. 2002). NGC 92 and NGC 88 are LINERs (Coziol et al. 2000) while NGC 89 is a Seyfert 2 galaxy (e.g. Pompei et al. 2007).

NGC 92 is classified as an Sa galaxy (Prugniel et al. 1998). It shows a prominent tidal tail in the south east direction also clearly visible in the $\mathrm{H} \alpha$ image of Temporin et al. (2005). The velocity field presented in Fig. 6 shows a disk-like rotation with a wide radial velocity range (from $3641 \mathrm{~km} \mathrm{~s}^{-1}$ to $3183 \mathrm{~km} \mathrm{~s}^{-1}$ ). The position angle of the velocity field changes from $8^{\circ}$ to $28^{\circ}$ over a 40 arcsec radius. Even with this strong change in the position angle, the velocity field is fairly regular. The center of the velocity field clearly shows double components. The second component is better defined in the blue part of the velocity field than in the red part, where it fades. Along the tail, this second component appears at the tip of the tail, but it is not possible to follow it all across the tail. Such double profiles have been observed in other asymmetric galaxies (e.g. UGC 3334, GHASP sample) and a tidal arm with an almost constant radial velocity has also been observed (e.g. UGC 3429, GHASP sample). The monochromatic map (Fig. 6) shows a very bright emission in the galaxy center. Much lower intensity emission regions are located in the northern region and across the tidal tail. These coincide with blobs also detected in the NUV-band image from GALEX (see Fig. 16). The central emission of NGC 92 shows a geometry consistent with spiral arms. There are a few hot spots along the tail also consistent with the GALEX image (see Fig. 16). The maximum velocity dispersion shown is $\sim 70 \mathrm{~km} \mathrm{~s}^{-1}$ but looking at the profiles it is obvious that several components are present. The dispersion map shows a higher velocity dispersion in the center of the galaxy than in its outskirts and tidal tail. In the center, the average velocity dispersion is $45 \mathrm{~km} \mathrm{~s}^{-1}$. Regions 1-3, detected with GALEX, have a lower velocity dispersion, less than $25 \mathrm{~km} \mathrm{~s}^{-1}$. The receding side reaches a rotational velocity of $243 \mathrm{~km} \mathrm{~s}^{-1}$ and the approaching side reaches $246 \mathrm{~km} \mathrm{~s}^{-1}$. The long tidal tail appears as an extension of the approaching side of the rotation curve, with a constant velocity of $160 \mathrm{~km} \mathrm{~s}^{-1}$ from 70 to $120^{\prime \prime}$. As HCG 7a, the rotation curve of NGC 92 presents a bump in velocity within $20^{\prime \prime}(5 \mathrm{kpc})$ that could be interpreted as a signature of non circular motion.

We also detected $\mathrm{H} \alpha$ emission at the center of NGC 88. However, we did not attempt to derive any rotation curve because this object is located at the edge of the image. However, we note that the velocity field appears uniform. 


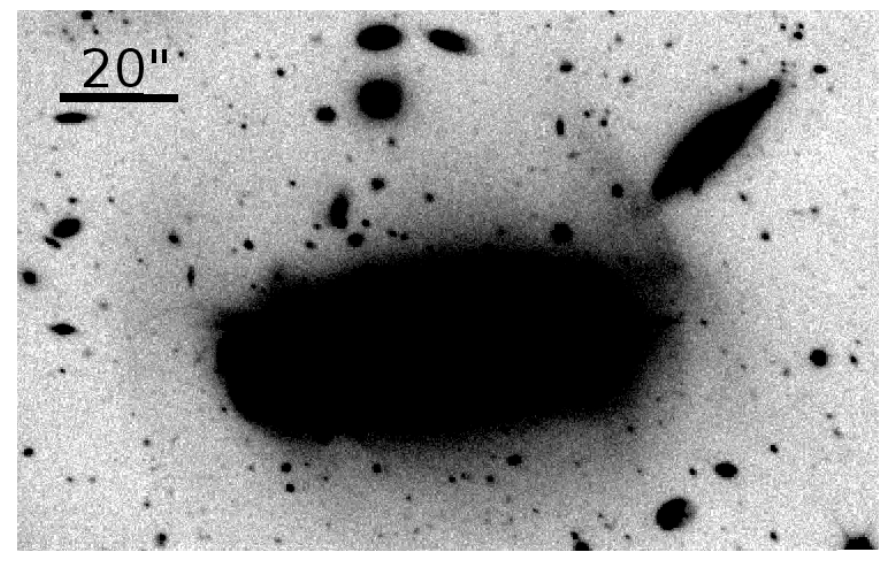

Fig. 9. $R$-band image of HCG 22b.

\subsection{Field density of UV emitting regions}

In Figs. 10 to 16 we show the GALEX NUV images for each compact group where sources are numbered in circles of 4 " radius. We cannot exclude the possibility that these regions are not related to the groups, given that we do not have redshift information for most of them. However, a similar search by de Mello et al. (2008a) using GALEX images of HCG 100 was able to detect two TDGs within the HI tail (which were spectroscopically confirmed by Urrutia-Viscarra et al. 2009). Moreover, de Mello et al. (2008b), using GALEX images, have detected star-forming regions in the vicinity of the HI bridge between M 81 and M 82 . Therefore, these two multiwavelength studies show that this approach is successful in identifying at least some star-forming regions in the intragroup medium. Determination of the membership and the true nature of these regions will have to await spectroscopy with large telescopes.

In order to evaluate whether our targets contain an excess of ultraviolet emitting regions in their vicinity, we have calculated the density of objects in the compact group field and in the control sample (CS). The field density was defined as the number of regions inside a circle of two group radii (see Sect. 3.2) divided by the area of that field after exclusion of the areas occupied by the galaxies, as described below. Regions detected inside the optical contours of group members (within $R_{25}$ ) were eliminated, since we are searching for star formation outside galaxies. This was done by discarding all regions inside an ellipse with major and minor axes $a$ and $b$, which correspond to the length of the major and minor axes of the $\mu_{B}=25 \mathrm{mag} \mathrm{arc}^{-2}$ isophote given in Hickson (1993). For galaxies HCG $92 \mathrm{~b}$ and d, we found it necessary to recompute the region within the $R_{25}$ isophote using the B image presented in Mendes de Oliveira et al. (2001), given the strong isophote overlap between these two systems. We note that three star-forming regions located over the tidal tail of NGC 92 were included in our analysis, given that these objects are located at projected distances outside the main body of this galaxy. We did not consider the area of each galaxy when estimating the total area of the group. No excess of ultraviolet emitting sources is detected in HCG 2, HCG 7, HCG 23 and NGC 92, when the groups are compared with the CS fields. Table 2 shows the spatial densities of objects around each compact group and in three control fields. HCG 92 contains the highest field density $\left(0.28 \pm 0.05\right.$ objects $\left.\operatorname{arcmin}^{-2}\right)$ in our sample. Most of the regions detected in this group are located in the tidal tails of NGC 7318A, NGC 7318B and NGC 7319. We detected UV emission in all intergalactic HII regions found by Mendes de Oliveira et al. (2004), confirming the results by

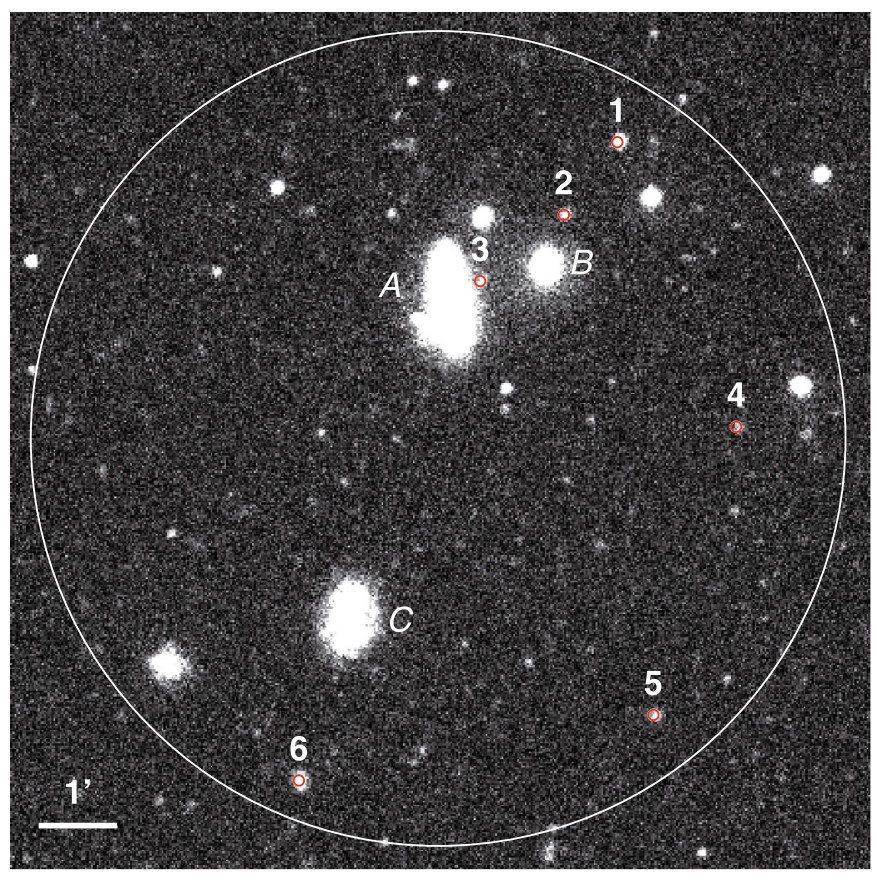

Fig. 10. NUV band image for HCG 2. The smaller circles show the detected regions. The large circle has a radius of twice the minimum circle that contains the galaxies members of the group, which are named with capital letters.

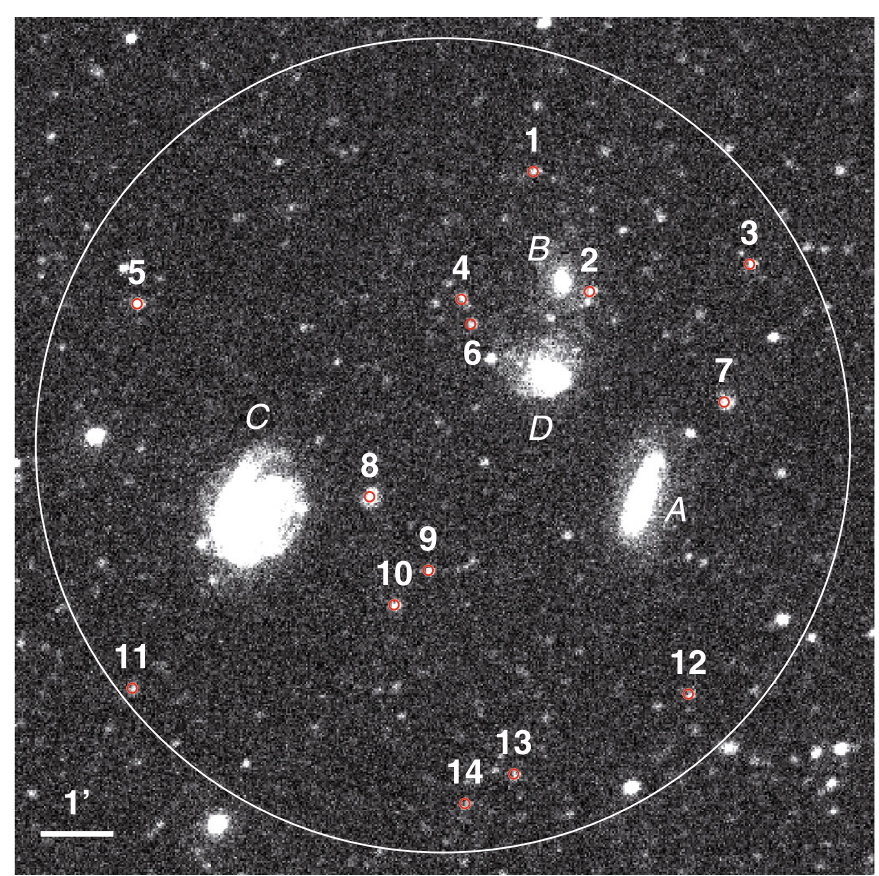

Fig. 11. NUV band image for HCG 7. See caption of Fig. 10.

Xu et al. (2005). Besides HCG 92, HCG 22 also has an excess of ultraviolet sources compared with their control sample. In this group, some of the UV detected regions show $8 \mu \mathrm{m}$ emission (Johnson et al. 2007). Although the field density of HCG 100 is slightly higher than in the CS fields, the error bars are large. Therefore, we have not found any significant excess of ultraviolet emitting regions in the intragroup field of HCG 100, when compared to the field density of the control sample. We note that the background counts are solely based on the observed counts in the control fields, not taking into account existing redshift 
Table 2. Field density $\Sigma$ (\#objects $\operatorname{arcmin}^{-2}$ )

\begin{tabular}{ccccc}
\hline \hline Compact group & $\Sigma$ Compact group & $\Sigma$ Control sample 1 & $\Sigma$ Control sample 2 & $\Sigma$ Control sample 3 \\
\hline HCG 2 & $0.07 \pm 0.03$ & $0.07 \pm 0.02$ & $0.05 \pm 0.02$ & $0.16 \pm 0.03$ \\
HCG 7 & $0.15 \pm 0.04$ & $0.16 \pm 0.03$ & $0.20 \pm 0.03$ & $0.17 \pm 0.03$ \\
HCG 22 & $0.25 \pm 0.07$ & $0.12 \pm 0.03$ & $0.11 \pm 0.03$ & $0.13 \pm 0.03$ \\
HCG 23 & $0.06 \pm 0.02$ & $0.08 \pm 0.02$ & $0.13 \pm 0.03$ & $0.11 \pm 0.03$ \\
HCG 92 & $0.28 \pm 0.05$ & $0.05 \pm 0.02$ & $0.08 \pm 0.02$ & $0.08 \pm 0.02$ \\
HCG 100 & $0.20 \pm 0.07$ & $0.09 \pm 0.02$ & $0.08 \pm 0.02$ & $0.14 \pm 0.03$ \\
NGC 92 & $0.16 \pm 0.07$ & $0.23 \pm 0.03$ & $0.20 \pm 0.03$ & $0.12 \pm 0.02$ \\
\hline
\end{tabular}

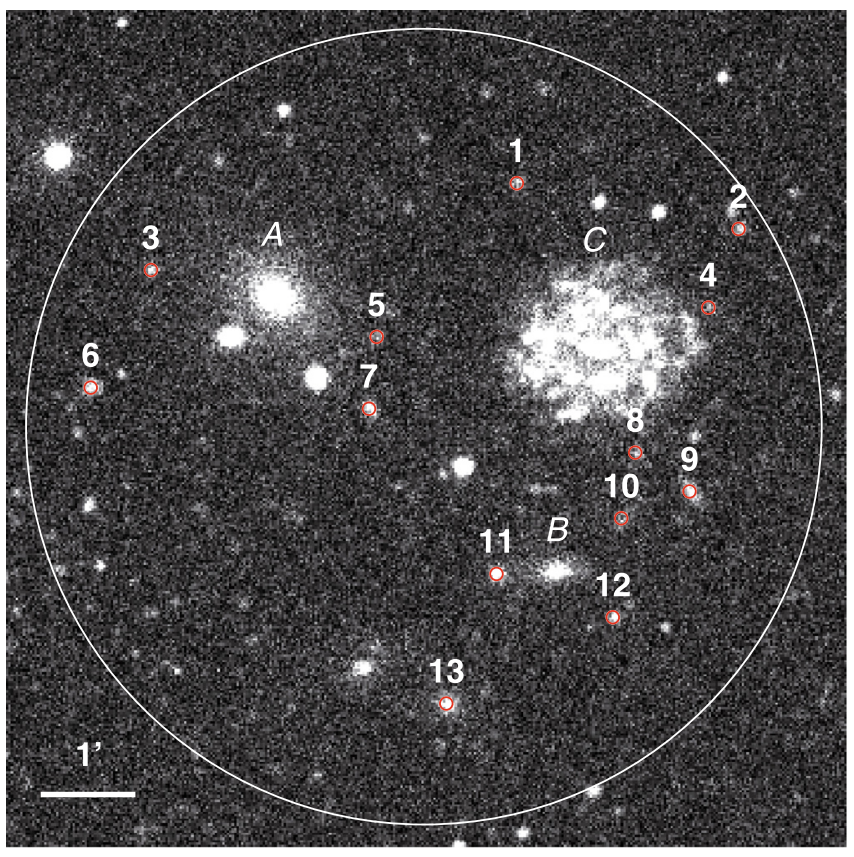

Fig. 12. NUV band image for HCG 22. See caption of Fig. 10.

information for individual regions, given that very few of them have known redshifts.

\subsection{FUV-NUV color distribution}

Since we are interested in young regions, we conservatively focused this analysis on objects bluer than FUV-NUV $=1$. We chose this limit based on the work of Gil de Paz et al. (2007) where it is reported that colors bluer than FUV-NUV $=0.9$ are typical of spiral and late-type galaxies. Objects with colors redder than FUV-NUV = 1 (see Figs. 18 and 19) account only for $6 \%$ of the total intragroup regions, $5 \%$ being stars (see Sect. 4.4). In the case of the control sample, objects with colors redder than FUV-NUV $=1$ correspond to $6 \%$ of the total. Tables 3 to 9 show the FUV-NUV colors for all detected regions in the compact group fields.

The FUV-NUV color distributions of the intragroup sources together with their respective CSs are shown in Fig. 17. We found that the CS color distributions are similar between themselves with the exception of that for HCG 23. We also found that only the intragroup regions of HCG 92 and HCG 100 show a blue FUV-NUV color distribution, as compared to the CS. HCG 92 has the bluest FUV-NUV average in this sample, FUV-NUV $=0.13 \pm 0.26$. Although the average in FUV-NUV color for HCG 100 is not among the bluest in this sample, FUV-NUV $=0.41 \pm 0.40$, the color distribution shows

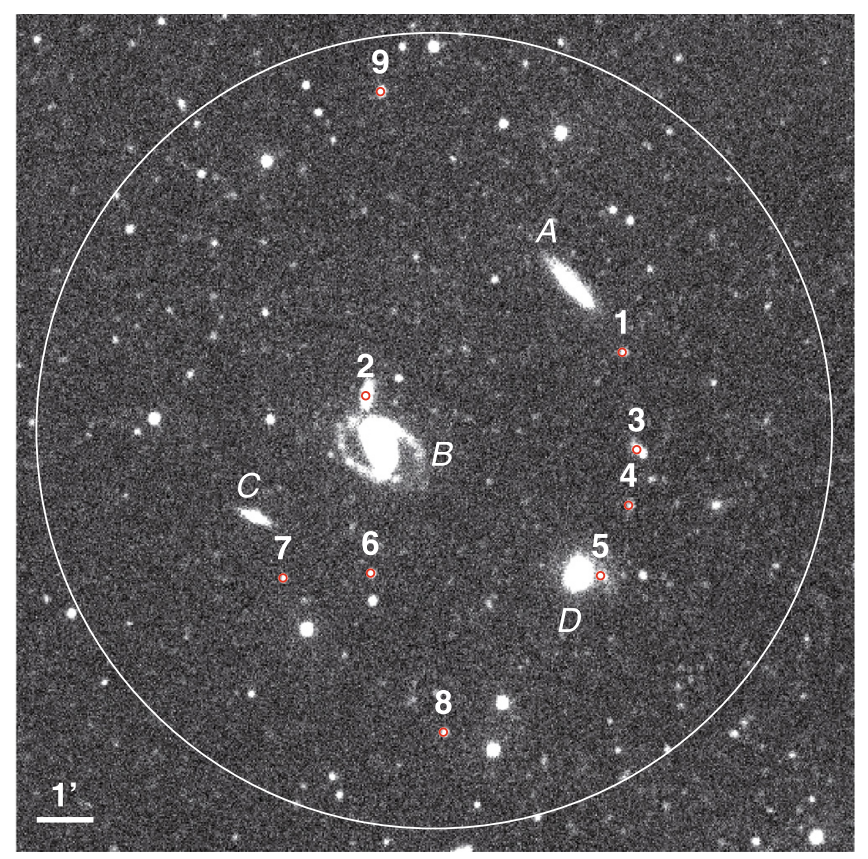

Fig. 13. NUV band image for HCG 23. See caption of Fig. 10.

a bimodal appearance, with a blue peak with a mean color of zero.

The intragroup color distribution is similar for HCG 7 and HCG 22. In both cases the intragroup FUV-NUV average is redder than their $\mathrm{CS}$, as shown in Fig. 17. From the same figure, HCG 2 shows a bimodal distribution, with a peak on the red side of the FUV-NUV color and a distribution similar to the control sample. In the case of HCG 23, the intragroup distribution shows no special signature compared to the control sample distribution. NGC 92 presents a single peak in the FUV-NUV distribution, with an average color equal to $0.28 \pm 0.15$. We note that NGC 92 contains only five detected intragroup regions, the lowest number in our sample.

HCG 7 has the reddest FUV-NUV average of all groups, $0.52 \pm 0.32$. Of a total of 14 regions, 4 regions $(29 \%$ of the intragroup sample) present colors bluer than the average FUV-NUV color for their control sample and 3 of them are located close to HCG 7c. Region \#5 is the bluest object in this compact group and shows stellar characteristics in the SDSS image. This object is $57.9 \mathrm{kpc}$ away from $\mathrm{HCG} 7 \mathrm{c}$.

The distribution of colors of intragroup objects in HCG 2 has an average FUV-NUV color of $0.47 \pm 0.38$. We found 3 regions $(50 \%$ of the intragroup sample) bluer than the average FUV-NUV of their control sample: region \#4, \#5 and \#6, located of $58.2 \mathrm{kpc}, 75.9 \mathrm{kpc}$ and $39.2 \mathrm{kpc}$ away from the closest galaxy (HCG 2b, HCG 2c and HCG 2c respectively). These objects show an extended morphology in our $R$-band 
Table 3. Observed and derived properties for HCG 2 regions.

\begin{tabular}{ccccccc}
\hline \hline ID & $\begin{array}{c}\text { RA } \\
(2000)\end{array}$ & $\begin{array}{c}\text { Dec } \\
(2000)\end{array}$ & FUV & NUV & FUV-NUV $^{a}$ & $\begin{array}{c}\text { Age }^{b} \\
(\mathrm{Myr})\end{array}$ \\
\hline 1 & 7.81289 & 8.50144 & $20.04 \pm 0.04$ & $19.04 \pm 0.02$ & $0.85 \pm 0.05$ & \\
2 & 7.82440 & 8.48604 & $21.46 \pm 0.10$ & $20.67 \pm 0.06$ & $0.49 \pm 0.11$ & \\
3 & 7.84238 & 8.47191 & $19.58 \pm 0.05$ & $18.73 \pm 0.02$ & $0.91 \pm 0.12$ & \\
4 & 7.78734 & 8.44088 & $21.99 \pm 0.14$ & $21.40 \pm 0.10$ & $0.33 \pm 0.14$ & \\
5 & 7.80517 & 8.37961 & $20.99 \pm 0.07$ & $20.85 \pm 0.06$ & $0.05 \pm 0.09$ & 40.8 \\
6 & 7.88140 & 8.36569 & $20.00 \pm 0.04$ & $19.72 \pm 0.03$ & $0.16 \pm 0.07$ & \\
\hline
\end{tabular}

${ }^{a}$ Using a fixed aperture of $4 " ;{ }^{b}$ age from FUV-NUV using Thilker et al. (2007).

Table 4. Observed and derived properties for HCG 7 regions.

\begin{tabular}{ccccccc}
\hline \hline ID & $\begin{array}{c}\text { RA } \\
(2000)\end{array}$ & $\begin{array}{c}\text { Dec } \\
(2000)\end{array}$ & FUV & NUV & FUV-NUV $^{a}$ & $\begin{array}{c}\text { Age }^{b} \\
(\mathrm{Myr})\end{array}$ \\
\hline 1 & 9.83047 & 0.93806 & $21.86 \pm 0.10$ & $21.41 \pm 0.09$ & $0.36 \pm 0.13$ & \\
2 & 9.81752 & 0.91055 & $21.83 \pm 0.11$ & $20.74 \pm 0.05$ & $0.90 \pm 0.13$ & \\
3 & 9.78083 & 0.91679 & $22.10 \pm 0.11$ & $21.09 \pm 0.06$ & $0.70 \pm 0.12$ & \\
4 & 9.84691 & 0.90873 & $21.81 \pm 0.10$ & $20.95 \pm 0.07$ & $0.65 \pm 0.14$ & \\
5 & 9.92126 & 0.90774 & $20.22 \pm 0.04$ & $20.23 \pm 0.03$ & $-0.14 \pm 0.05$ & 3.6 \\
6 & 9.84480 & 0.90307 & $22.48 \pm 0.13$ & $21.18 \pm 0.08$ & $0.93 \pm 0.16$ & \\
7 & 9.78668 & 0.88524 & $21.38 \pm 0.08$ & $20.35 \pm 0.04$ & $0.71 \pm 0.11$ & \\
8 & 9.86793 & 0.86351 & $20.29 \pm 0.05$ & $19.79 \pm 0.03$ & $0.49 \pm 0.08$ & \\
9 & 9.85452 & 0.84667 & $22.33 \pm 0.12$ & $21.33 \pm 0.06$ & $0.76 \pm 0.13$ & \\
10 & 9.86234 & 0.83872 & $21.24 \pm 0.07$ & $20.89 \pm 0.05$ & $0.27 \pm 0.09$ & \\
11 & 9.92238 & 0.81964 & $21.82 \pm 0.09$ & $21.76 \pm 0.08$ & $-0.05 \pm 0.12$ & 12.2 \\
12 & 9.79487 & 0.81829 & $22.39 \pm 0.13$ & $21.61 \pm 0.09$ & $0.43 \pm 0.14$ & \\
13 & 9.83494 & 0.79998 & $22.46 \pm 0.13$ & $21.55 \pm 0.09$ & $0.75 \pm 0.16$ & \\
14 & 9.84611 & 0.79318 & $22.46 \pm 0.14$ & $21.65 \pm 0.11$ & $0.46 \pm 0.17$ & \\
\hline
\end{tabular}

${ }^{a}$ Using a fixed aperture of $4 "{ }^{\prime}{ }^{b}$ age from FUV-NUV using Thilker et al. (2007).

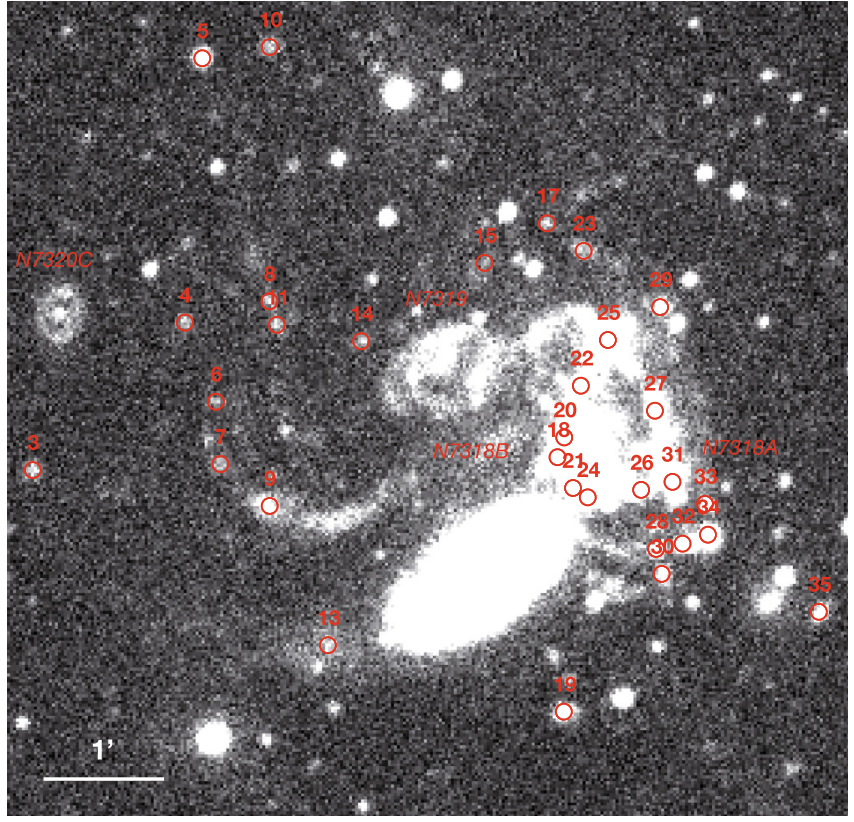

Fig. 14. Zoom of the central region of HCG 92 (NUV band image). See caption of Fig. 10. Galaxy names were taken from Mendes de Oliveira et al. (2004).

image. Regions \#2 and \#3 seem to be associated with the regions identified in the monochromatic map of HCG 2, as we noted in Sect. 4.1.1.

In the case of HCG 22, the objects in the intragroup medium have an average FUV-NUV color of $0.32 \pm 0.26$ and this group

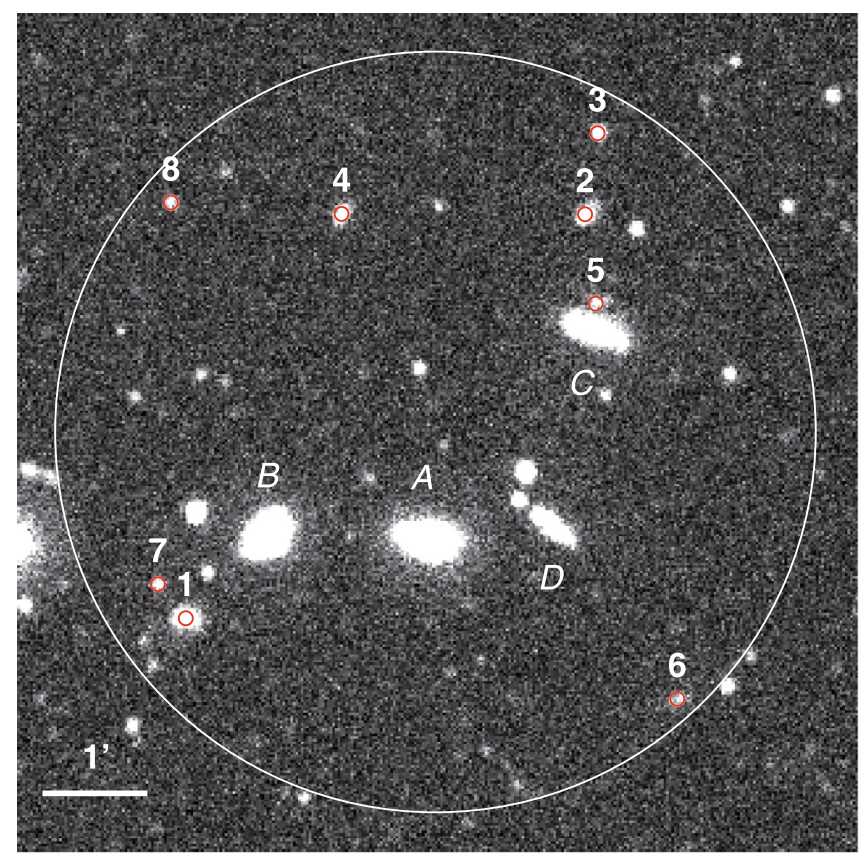

Fig. 15. NUV band image for HCG 100. See caption of Fig. 10.

contains 6 regions ( $46 \%$ of the intragroup sample) bluer than the average color of the control sample counterparts.

For HCG 23, the FUV-NUV average is $0.36 \pm 0.28$, with 3 regions (33\% of the intragroup sample) being bluer than their control sample. 
Table 5. Observed and derived properties for HCG 22 regions

\begin{tabular}{cccccccc}
\hline \hline ID & $\begin{array}{c}\text { RA } \\
(2000)\end{array}$ & $\begin{array}{c}\text { Dec } \\
(2000)\end{array}$ & FUV & NUV & FUV-NUV $^{a}$ & $\begin{array}{c}\text { Age }^{b} \\
(\mathrm{Myr})\end{array}$ & $\begin{array}{c}\text { Velocity }^{c} \\
\left(\mathrm{~km} \mathrm{~s}^{-1}\right)\end{array}$ \\
\hline 1 & 45.86570 & -15.59343 & $22.04 \pm 0.19$ & $21.45 \pm 0.09$ & $0.57 \pm 0.20$ & & \\
2 & 45.82517 & -15.60148 & $21.79 \pm 0.15$ & $21.39 \pm 0.09$ & $0.10 \pm 0.16$ & 60.8 & \\
3 & 45.93254 & -15.60869 & $21.57 \pm 0.14$ & $21.31 \pm 0.09$ & $-0.05 \pm 0.16$ & 12.2 & \\
4 & 45.83071 & -15.61531 & $21.93 \pm 0.18$ & $21.27 \pm 0.10$ & $-0.05 \pm 0.20$ & 12.2 & \\
5 & 45.89130 & -15.62050 & $22.07 \pm 0.18$ & $21.63 \pm 0.12$ & $0.04 \pm 0.20$ & 37.3 & \\
6 & 45.94355 & -15.62937 & $21.15 \pm 0.10$ & $20.59 \pm 0.06$ & $0.20 \pm 0.13$ & & \\
7 & 45.89273 & -15.63312 & $21.42 \pm 0.14$ & $20.59 \pm 0.06$ & $0.52 \pm 0.16$ & & \\
8 & 45.84404 & -15.64084 & $21.85 \pm 0.17$ & $21.11 \pm 0.09$ & $0.26 \pm 0.20$ & & 29108 \\
9 & 45.83414 & -15.64766 & $20.70 \pm 0.10$ & $20.26 \pm 0.05$ & $0.35 \pm 0.15$ & & \\
10 & 45.84662 & -15.65240 & $22.24 \pm 0.20$ & $20.90 \pm 0.09$ & $0.54 \pm 0.25$ & & \\
11 & 45.86941 & -15.66216 & $21.13 \pm 0.11$ & $20.08 \pm 0.04$ & $0.58 \pm 0.13$ & & \\
12 & 45.84815 & -15.66981 & $21.60 \pm 0.14$ & $21.01 \pm 0.07$ & $0.37 \pm 0.15$ & & 9342 \\
13 & 45.87857 & -15.68500 & $21.29 \pm 0.12$ & $19.76 \pm 0.04$ & $0.72 \pm 0.15$ & & \\
\hline
\end{tabular}

${ }^{a}$ Using a fixed aperture of $4 "{ }^{\prime \prime}{ }^{b}$ age from FUV-NUV using Thilker et al. (2007); ${ }^{c}$ velocity taken from de Carvalho et al. (1997) and Hickson et al. (1992) for object 9 and 13 respectively.

Table 6. Observed and derived properties for HCG 23 regions.

\begin{tabular}{cccccccc}
\hline \hline ID & $\begin{array}{c}\text { RA } \\
(2000)\end{array}$ & $\begin{array}{c}\text { Dec } \\
(2000)\end{array}$ & FUV & NUV & FUV-NUV $^{a}$ & $\begin{array}{c}\text { Age }^{b} \\
(\mathrm{Myr})\end{array}$ & $\begin{array}{c}\text { Velocity }^{c} \\
\left(\mathrm{~km} \mathrm{~s}^{-1}\right)\end{array}$ \\
\hline 1 & 46.71703 & -9.56431 & $21.64 \pm 0.11$ & $21.02 \pm 0.05$ & $0.50 \pm 0.10$ & & \\
2 & 46.79337 & -9.57706 & $18.67 \pm 0.03$ & $17.86 \pm 0.01$ & $0.49 \pm 0.05$ & & 10150 \\
3 & 46.71273 & -9.59288 & $19.81 \pm 0.04$ & $19.51 \pm 0.02$ & $0.41 \pm 0.07$ & & \\
4 & 46.71515 & -9.60920 & $21.55 \pm 0.14$ & $21.24 \pm 0.07$ & $0.45 \pm 0.13$ & & \\
5 & 46.72348 & -9.62984 & $20.49 \pm 0.07$ & $19.31 \pm 0.03$ & $0.45 \pm 0.08$ & & \\
6 & 46.79189 & -9.62909 & $21.64 \pm 0.10$ & $20.56 \pm 0.03$ & $0.82 \pm 0.09$ & & \\
7 & 46.81795 & -9.63047 & $21.30 \pm 0.09$ & $21.29 \pm 0.06$ & $-0.07 \pm 0.09$ & 10.0 & \\
8 & 46.77020 & -9.67570 & $21.11 \pm 0.09$ & $20.87 \pm 0.04$ & $0.20 \pm 0.08$ & & \\
9 & 46.78895 & -9.48790 & $19.90 \pm 0.05$ & $19.90 \pm 0.02$ & $0.00 \pm 0.05$ & 21.5 & 5283 \\
\hline
\end{tabular}

${ }^{a}$ Using a fixed aperture of $4 "{ }^{\prime}{ }^{b}$ age from FUV-NUV using Thilker et al. (2007); ${ }^{c}$ velocity taken from de Carvalho et al. (1997) and Hickson et al. (1992) for object 9 and 2 respectively.

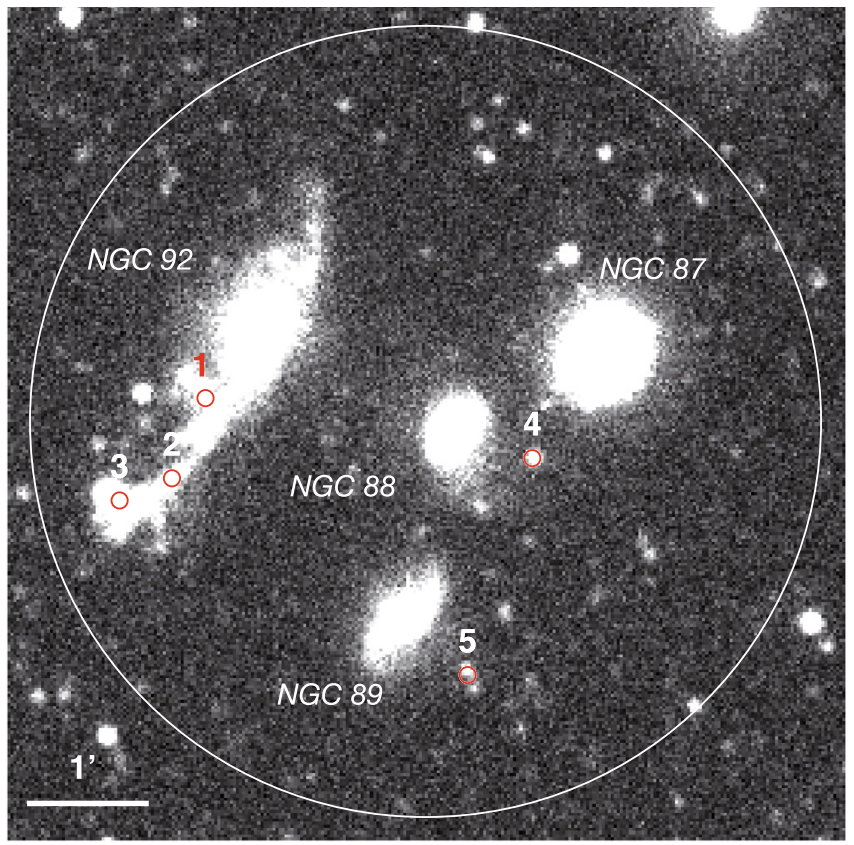

Fig. 16. NUV band image for NGC 92. See caption of Fig. 10.

As we noted before, HCG 92 has the bluest FUV-NUV average and distribution. In this group, $78 \%$ of the intragroup candidates (28 regions) are bluer than the value for the FUV-NUV control sample average, mainly placed in the tidal tails, as has been reported in Mendes de Oliveira et al. (2001). The regions in common between that work and ours are indicated in the second column of Table 7 .

In $\mathrm{HCG} 100$, we found 4 regions (50\% of the intragroup sample) bluer than the average values of the CS FUV-NUV: regions \#4 (\#4), \#6 (\#6), \#7 (\#13) and \#8 (\#14). Note that our identification in Table 8 is not the same as in de Mello et al. (2008a), the latter are given in parentheses.

In NGC 92, $60 \%$ of the regions are bluer than the average color of the regions of the control sample. Interestingly, the bluest regions in this group, \#1, \#2 and \#3, are located in the tidal tail of NGC 92, where it is possible to note a gradient in the FUV-NUV color along the tail, with the bluest region located at the end of the tidal tail. Similar effects were reported by Hibbard et al. (2005) for NGC 4038/39.

Considering the relatively small size of our samples and large dispersions in the averages, we have to be particularly careful in our analysis. Therefore, instead of comparing average numbers, we performed the Kolmogorov-Smirnov (KS) statistical test comparing the color distribution of each group with its corresponding control sample (Table 10). The most significant result is for HCG 92, where the intragroup FUV-NUV color distribution shows only a $0.001 \%$ probability of being drawn from the control sample distribution. For the other groups, HCG 2, HCG 22, and NGC 92 have distributions similar to their control samples, at the $67 \%, 47 \%$, and $96 \%$ level, respectively. HCG 7 and HCG 100, on the other hand, are similar only at $6 \%$ and 
Table 7. Observed and derived properties for HCG 92 regions.

\begin{tabular}{|c|c|c|c|c|c|c|}
\hline ID & $\mathrm{ID}^{a}$ & $\begin{array}{c}\text { RA } \\
(2000) \\
\end{array}$ & $\begin{array}{c}\text { Dec } \\
(2000) \\
\end{array}$ & FUV-NUV $^{b}$ & $\begin{array}{l}\mathrm{Age}^{c} \\
(\mathrm{Myr})\end{array}$ & $\begin{array}{l}\text { Velocity }{ }^{d} \\
\left(\mathrm{~km} \mathrm{~s}^{-1}\right)\end{array}$ \\
\hline 1 & $\ldots$ & 339.11306 & 34.03644 & $-0.09 \pm 0.08$ & 5.1 & \\
\hline 2 & $\ldots$ & 339.10057 & 34.04839 & $0.73 \pm 0.12$ & & \\
\hline 3 & $\ldots$ & 339.08941 & 33.96354 & $0.53 \pm 0.13$ & & \\
\hline 4 & $\mathrm{a}$ & 339.06386 & 33.98405 & $-0.16 \pm 0.10$ & 3.4 & 6600 \\
\hline 5 & & 339.06090 & 34.02086 & $-0.56 \pm 0.03$ & 0.1 & \\
\hline 6 & $\mathrm{~b}$ & 339.05867 & 33.97303 & $-0.12 \pm 0.13$ & 3.9 & 6651 \\
\hline 7 & $\ldots$ & 339.05790 & 33.96437 & $-0.09 \pm 0.11$ & 5.1 & \\
\hline 8 & d & 339.04965 & 33.98702 & $-0.18 \pm 0.10$ & 3.2 & 6616 \\
\hline 9 & $\ldots$ & 339.04965 & 33.95852 & $0.20 \pm 0.07$ & & \\
\hline 10 & $\ldots$ & 339.04957 & 34.02240 & $0.20 \pm 0.12$ & & \\
\hline 11 & $\mathrm{c}$ & 339.04842 & 33.98374 & $0.30 \pm 0.12$ & & 6565 \\
\hline 12 & $\ldots$ & 339.04518 & 34.03890 & $0.37 \pm 0.11$ & & \\
\hline 13 & $\ldots$ & 339.03988 & 33.93911 & $0.47 \pm 0.11$ & & \\
\hline 14 & $\ldots$ & 339.03426 & 33.98144 & $0.21 \pm 0.13$ & & \\
\hline 15 & $\ldots$ & 339.01353 & 33.99232 & $0.00 \pm 0.13$ & 21.5 & \\
\hline 16 & $\ldots$ & 339.00492 & 33.89762 & $0.41 \pm 0.07$ & & \\
\hline 17 & $\ldots$ & 339.00299 & 33.99783 & $-0.2 \pm 0.10$ & 3.1 & \\
\hline 18 & 10 & 339.00132 & 33.96527 & $0.14 \pm 0.04$ & & \\
\hline 19 & $\ldots$ & 339.00029 & 33.92982 & $0.49 \pm 0.07$ & & \\
\hline 20 & $\ldots$ & 339.00018 & 33.96802 & $0.19 \pm 0.04$ & & \\
\hline 21 & 12 & 338.99871 & 33.96096 & $0.2 \pm 0.04$ & & \\
\hline 22 & 7 & 338.99737 & 33.97522 & $0.16 \pm 0.05$ & & \\
\hline 23 & $\ldots$ & 338.99687 & 33.99399 & $0.04 \pm 0.10$ & 37.3 & \\
\hline 24 & 14 & 338.99626 & 33.95968 & $0.13 \pm 0.03$ & & \\
\hline 25 & 13 & 338.99283 & 33.98158 & $0.02 \pm 0.03$ & 30.5 & \\
\hline 26 & 5 & 338.98729 & 33.96068 & $0.11 \pm 0.05$ & & \\
\hline 27 & 16 & 338.98496 & 33.97171 & $0.05 \pm 0.04$ & 40.8 & \\
\hline 28 & 8 & 338.98478 & 33.95249 & $-0.07 \pm 0.07$ & 10.0 & \\
\hline 29 & 23 & 338.98404 & 33.98615 & $0.25 \pm 0.08$ & & \\
\hline 30 & $\ldots$ & 338.98385 & 33.94899 & $-0.02 \pm 0.08$ & 19.1 & \\
\hline 31 & $\ldots$ & 338.98203 & 33.96181 & $0.00 \pm 0.03$ & 21.5 & \\
\hline 32 & 18 & 338.98033 & 33.95322 & $-0.11 \pm 0.05$ & 4.0 & \\
\hline 33 & 22 & 338.97658 & 33.95873 & $0.23 \pm 0.08$ & & \\
\hline 34 & 20 & 338.97609 & 33.95446 & $-0.02 \pm 0.04$ & 19.1 & \\
\hline 35 & 21 & 338.95747 & 33.94368 & $0.55 \pm 0.09$ & & \\
\hline 36 & $\ldots$ & 338.94114 & 33.91203 & $0.44 \pm 0.11$ & & \\
\hline
\end{tabular}

${ }^{a}$ ID in Mendes de Oliveira et al. (2001, 2004); ${ }^{b}$ using a fixed aperture of $4 "{ }^{\prime \prime}{ }^{c}$ age from FUV-NUV using Thilker et al. (2007); ${ }^{d}$ taken from Mendes de Oliveira et al. (2004).

$33 \%$ levels respectively (note that for HCG 7 regions are on average redder than the $\mathrm{CS}$ regions). If we take the intragroup sample of de Mello et al. (2008a) for HCG 100 and its respective control sample, we find only a $6 \%$ probability, which confirms that de Mello et al. (2008a) intragroup regions are significantly bluer than regions outside the group.

In order to increase our control sample and improve our statistics, we generated one total control sample by adding all control samples (Table 10). The average UV color for the total control sample is FUV-NUV $=0.35 \pm 0.25$. This value is slightly lower than the peak in the FUV-NUV color distribution given in Gil de Paz et al. (2007) (FUV-NUV $\simeq 0.4$ ) but still consistent with it. This small difference is due to our criterios of colors bluer than FUV-NUV $=1$. When we consider the control sample in the same range of colors as Gil de Paz et al. (2007) $(-0.4 \leq \mathrm{FUV}-\mathrm{NUV} \leq 3.2)$, our average is FUV-NUV $=0.4$.

HCG 92, HCG 22 and NGC 92 have FUV-NUV averages FUV-NUV $=0.13 \pm 0.26$, FUV-NUV $=0.32 \pm 0.26$, FUV-NUV $=0.28 \pm 0.15$ bluer than the total control sample $(\mathrm{FUV}-\mathrm{NUV}=0.35 \pm 0.25)$. When we compare each group sample with the total CS, the KS test gives the lowest probabilities for HCG 92 ( 0\%), HCG 7 (3\%), and HCG 100 (8\% or 32\% depending if we use de Mello et al. (2008a) or the HCG 100 generated in this work).

Finally, we added all regions of all groups and compared the FUV-NUV distribution with that of the total control sample. We find a probability of $0.005 \%$ that CS and group regions are drawn from the same distribution of colors. However, when we remove from the list all regions belonging to HCG 92 and HCG 100, the probability increases to $48 \%$. Therefore, we conclude that this is clear evidence that these two groups are significantly different from the other five in this respect. Moreover, as shown in Sect. 5.3, these two groups are also unique in their HI properties.

\subsection{Colors, photometric ages and SFR}

In Fig. 18 we plot FUV-NUV and FUV- $R$ colors of the intragroup candidates (colors estimated inside a fixed aperture of 4 " radius) and the stellar population synthesis models given in Thilker et al. (2007). These models are tuned for GALEX and optical colors, using an instantaneous burst, a solar metallicity and Chabrier IMF. We plot models assuming intrinsic colors and reddened colors for $E(B-V)=0.2$ and a Milky Way attenuation law $\left(R_{V}=3.1\right)$. We note that bluer objects than FUV-NUV $\leq 0.25$ are located close to the Milky Way attenuation law. On the other hand, objects redder than this value are mostly placed between the models with a Milky Way extinction law and with no extinction. Gil de Paz et al. (2007), using more than one thousand galaxies, suggested that the attenuation law in the UV is different from a pure Galactic extinction law (Fig. 8b in Gil de Paz et al. 2007). From this fact and from Fig. 18, we conservatively estimated ages of all objects with FUV-NUV $<0.1$ using Thilker et al. (2007) with Milky Way extinction corrections. Tables 3 to 9 show the ages for each region. In Fig. 18, we show the intergalactic HII regions (IHII) detected in Mendes de Oliveira et al. (2004) where we can see that two of the four spectroscopically confirmed IHII regions can be adjusted by the Milky Way extinction law.

For each region with known distance, we estimated the Star Formation Rate (SFR) using the ultraviolet luminosity of each object. In order to correct luminosities for the dust effect, we used our FUV-NUV colors to obtain the total-infrared to FUV ratio (IR/FUV) for each galaxy using Eq. (11) of Gil de Paz et al. (2007), since we do not have infrared data for our galaxies. The $A_{\mathrm{FUV}}$ value was then obtained inserting the derived value of IR/FUV, estimated as above, in the Eq. (2) of Buat et al. (2005). The UV luminosity is a tracer of star formation since most of the UV photons coming from a galaxy are produced by young stars. In this case, the SFR is proportional to the UV luminosity emitted by OB stars, under the assumption that the SFR is approximately constant over this timescale (Iglesias-Páramo et al. 2006). The SFR was estimated (Table 9) using the equations given by Iglesias-Páramo et al. (2006) and the FUV luminosities for each region. Note that the dispersion found in Eq. (11) of Gil de Paz et al. (2007) is quite high (0.36 dex), and therefore this introduces large uncertainties in the derived values of the UV attenuation and consequently in the determined values of SFRs (listed only for three regions with known redshifts for group NGC 92, see Table 9).

Figure 19 shows the NUV- $R$ versus FUV-NUV plot for all regions where we also include objects with colors redder than FUV-NUV $=1$. The reddest object in HCG 2 is outside the region populated by early-type objects (defined by Gil de Paz et al. 2007) and shows a stellar morphology in our $R$-band image. Two of the HCG 7 sources also show a stellar profile in the $r$-band image, therefore, only one object with color FUV-NUV $\geq 1$ shows 
Table 8. Observed and derived properties for HCG 100 regions.

\begin{tabular}{cccccccc}
\hline \hline ID & ID $^{a}$ & $\begin{array}{c}\text { RA } \\
(2000)\end{array}$ & $\begin{array}{c}\text { Dec } \\
(2000)\end{array}$ & FUV & NUV & FUV-NUV $^{b}$ & $\begin{array}{c}\text { Age }^{c} \\
(\mathrm{Myr})\end{array}$ \\
\hline 1 & $\ldots$ & 0.37526 & 13.16465 & $21.90 \pm 0.10$ & $21.38 \pm 0.06$ & $0.47 \pm 0.10$ & \\
2 & $\ldots$ & 0.30563 & 13.17565 & $21.70 \pm 0.08$ & $20.29 \pm 0.03$ & $0.81 \pm 0.07$ & \\
3 & $\ldots$ & 0.34740 & 13.16286 & $21.34 \pm 0.07$ & $20.31 \pm 0.04$ & $0.82 \pm 0.09$ & \\
$4^{d}$ & $4^{d}$ & 0.29263 & 13.08581 & $21.39 \pm 0.09$ & $20.95 \pm 0.07$ & $0.00 \pm 0.10$ & 21.49 \\
5 & $\ldots$ & 0.30592 & 13.14859 & $21.79 \pm 0.11$ & $20.70 \pm 0.06$ & $0.91 \pm 0.12$ & \\
6 & 6 & 0.30766 & 13.16278 & $20.38 \pm 0.05$ & $19.92 \pm 0.03$ & $0.23 \pm 0.06$ & \\
7 & 13 & 0.37282 & 13.09862 & $19.65 \pm 0.03$ & $19.19 \pm 0.02$ & $0.07 \pm 0.05$ & 48.75 \\
8 & 14 & 0.37734 & 13.10402 & $21.51 \pm 0.08$ & $21.12 \pm 0.07$ & $-0.06 \pm 0.08$ & 10.86 \\
$\ldots$ & $3^{d}$ & 0.27220 & 13.02360 & $21.21 \pm 0.09$ & $21.61 \pm 0.08$ & $-0.40 \pm 0.12$ & $<1$ \\
\hline
\end{tabular}

${ }^{a}$ ID in de Mello et al. (2008a); ${ }^{b}$ using a fixed aperture of $4{ }^{\prime \prime} ;{ }^{c}$ age from FUV-NUV using Thilker et al. (2007); ${ }^{d}$ recently obtained spectroscopy confirms these regions as part of HCG 100 (Urrutia-Viscarra et al. 2009).

Table 9. Observed and derived properties for NGC 92 regions.

\begin{tabular}{|c|c|c|c|c|c|c|c|c|}
\hline ID & $\begin{array}{c}\text { RA } \\
(2000)\end{array}$ & $\begin{array}{c}\text { Dec } \\
(2000)\end{array}$ & FUV & NUV & FUV-NUV $^{a}$ & $\begin{array}{l}\text { Age }^{b} \\
\text { (Myr) }\end{array}$ & $\begin{array}{c}S F R_{\mathrm{FUV}}{ }^{c} \\
\left(M_{\odot} \mathrm{yr}^{-1}\right)\end{array}$ & $\begin{array}{c}\text { Velocity } \\
\left(\mathrm{km} \mathrm{s}^{-1}\right)^{d}\end{array}$ \\
\hline 1 & 5.39251 & -48.63508 & $20.04 \pm 0.03$ & $19.67 \pm 0.02$ & $0.34 \pm 0.07$ & & 0.008 & 3283 \\
\hline 2 & 5.39949 & -48.64607 & $20.55 \pm 0.04$ & $20.31 \pm 0.01$ & $0.19 \pm 0.06$ & & 0.003 & 3289 \\
\hline 3 & 5.41036 & -48.64908 & $18.96 \pm 0.02$ & $18.77 \pm 0.01$ & $0.06 \pm 0.04$ & 44.6 & 0.011 & 3294 \\
\hline 4 & 5.32479 & -48.64329 & $21.38 \pm 0.07$ & $19.99 \pm 0.03$ & $0.43 \pm 0.08$ & & & \\
\hline 5 & 5.33817 & -48.67298 & $21.75 \pm 0.10$ & $21.07 \pm 0.05$ & $0.38 \pm 0.12$ & & & \\
\hline
\end{tabular}

${ }^{a}$ Using a fixed aperture of $4 "$.

${ }^{b}$ Age from FUV-NUV using Thilker et al. (2007).

${ }^{c} S F R\left(M_{\odot} \mathrm{yr}^{-1}\right)$ from Iglesias-Páramo et al. (2006) using FUV corrected for internal extinction from Gil de Paz et al. (2007) and Buat et al. (2005).

Note that these values have uncertainties of typically a factor of 2 , given that we have no measured IR/UV ratios for these objects.

${ }^{d}$ Estimated from the velocity field.

Table 10. Statistical values.

\begin{tabular}{ccccc}
\hline \hline & \multicolumn{2}{c}{ Each control sample } & \multicolumn{2}{c}{ Total control sample } \\
& $D^{a}$ & $P^{b}$ & $D$ & $P$ \\
\hline HCG 2 & 0.29 & 0.67 & 0.30 & 0.56 \\
HCG 7 & 0.36 & 0.06 & 0.37 & 0.03 \\
HCG 22 & 0.25 & 0.47 & 0.18 & 0.79 \\
HCG 23 & 0.31 & 0.39 & 0.29 & 0.39 \\
HCG 92 & 0.40 & 0.00 & 0.42 & 0.00 \\
HCG 100 & 0.33 & 0.33 & 0.32 & 0.32 \\
HCG 100 & 0.35 & 0.06 & 0.31 & 0.08 \\
NGC 92 & 0.21 & 0.96 & 0.22 & 0.95 \\
All Regions & \\
All Regions $^{e}$ & & & 0.20 & 0.00 \\
\hline
\end{tabular}

${ }^{a} D$ is the maximum vertical deviation between two cumulative distribution in the K-S statistics.

${ }^{b} P$ gives the significance level of the K-S statistic. Small values of $P$ show that the cumulative distribution function of CS is significantly different from intragroup regions.

${ }^{c}$ Colors of HCG 100 objects from de Mello et al. (2008a) were estimated using fixed aperture.

${ }^{d}$ Using HCG 100 detected regions from this work.

${ }^{e}$ Using HCG 100 detected regions from de Mello et al. (2008a).

an extended morphology. In the case of HCG 92, two objects with colors redder than FUV-NUV $=1$ resemble stars (these are objects without $R$-band information). Another 4 objects in the plot show colors slightly redder than FUV-NUV $=0.9$, which is the limit defined by Gil de Paz et al. (2007) as typical of earlytype galaxies. However, considering the relatively large error bars they could also be classified as spiral galaxies. All other intragroup candidates have colors typical of late-type galaxies.

\section{Discussion}

\subsection{Kinematics and the evolutionary stage of each group}

We used the scheme devised by Amram et al. (2003) and Plana et al. (2003) to classify galaxies for which we have Fabry-Perot velocity maps as interacting or not, based on the analysis of their $2 \mathrm{D}$ velocity fields and the determination of seven interaction indicators. The first indicator of interactions is the presence of strong peculiarities in the velocity field, which may display noncircular motion. A highly peculiar velocity field, not common for normal galaxies (see e.g. Epinat et al. 2008a,b), is a strong sign of interaction. A perturbed velocity field leads, by consequence, to a peculiar rotation curve, except if the asymmetries seen in the velocity field are smoothed by azimuthal averaging. This is the case, for example, for HCG 7a which shows an asymmetric velocity field with respect to the major axis (Fig. 3), completely smoothed on the rotation curve after the azimuthal averaging (Fig. 7d). On the other hand, asymmetries with respect to the minor axis of the velocity field are easier to notice by comparing both sides of the rotation curve than by inspecting the velocity field. Therefore, we introduce this specific interaction indicator which refers to asymmetries in the shapes of the rotation curves. A rotation curve is considered peculiar when it is not possible to match the shape of the receding with that of the approaching side, no matter how one chooses the galaxy kinematic center and systemic velocity.

The third and fourth indicators refer to the orientation of the PA of the galaxy. Epinat et al. (2008b) showed that $21 \%$ (33 objects) of field late-type galaxies from the GHASP survey have a misalignment between kinematic and morphological axes greater than $20^{\circ}$. From these 33 , if we remove galaxies with low inclinations and with poor morphological PA 

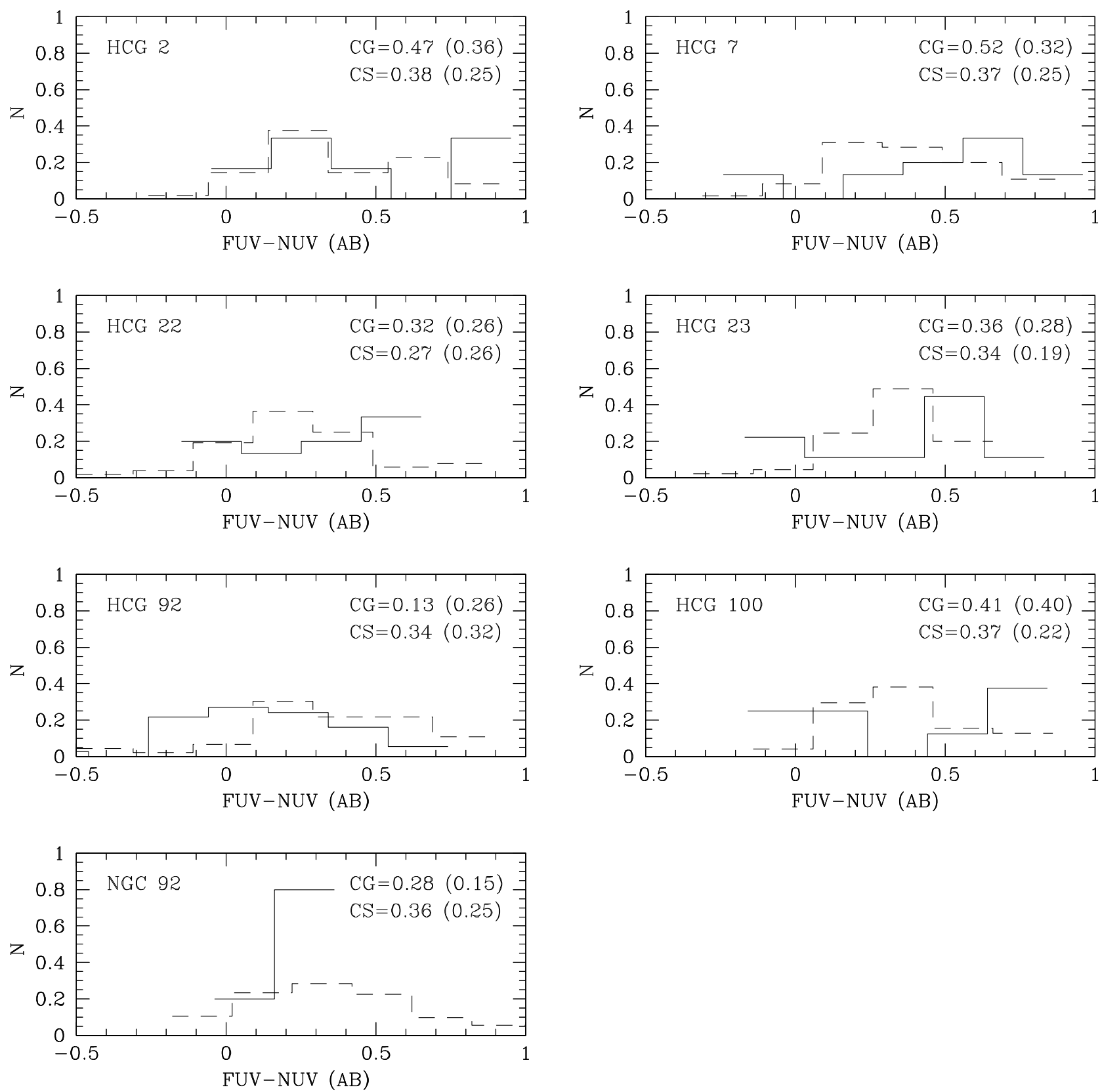

Fig. 17. Normalized histograms showing FUV-NUV distributions. Solid line: intragroup regions. Dashed line: control sample. Averages for each FUV-NUV color distribution are shown as CG, for compact group and CS, for control sample. Values for the standard deviation are indicated in parentheses. The error bar in the $x$-axis is estimated to be 0.2 mag.

determinations, almost all remaining ones have bars. This means that for field galaxies (as represented by the GHASP sample), the misalignment between stellar and kinematic axes happens quite rarely, except for barred galaxies. The presence of a bar has not been proven observationally to be directly associated with interactions, although several $N$-body simulation studies of bar formation have suggested that a connection between galaxy-galaxy interaction and bar formation exists and depends on the orbital parameters of the encounter (e.g. Debattista et al. 2006; Gerin et al. 1990a). On the other hand, it has been observed that in several non-barred interacting galaxies, there is a clear misalignment between the gas and stellar PA or a change of PA along the major axes, given that accreted gas can settle in a different disk (e.g. galaxies in HCG 16, Mendes de Oliveira et al. 1998; and in HCG 100, Plana et al. 2003). For this reason we include "gaseous versus stellar major-axis misalignment" and "changing position angle along major axis" as interaction indicators in Table 11, although we have to keep in mind that these indicators may also be related to the existence of a bar (such cases are flagged in Table 11 with footnote b).

Another indicator is the presence of multiple components in the $\mathrm{H} \alpha$ profiles. It is used to show evidence for strong interactions or mergers although such profiles could, in general, also be associated with the presence of an AGN. Finally, the last indicators are related to the existence of tidal tails, if the galaxy has high IR emission or not and the presence of central activity for each galaxy. A list with these parameters for all galaxies studied here is given in Table 11. The first four indicators for members of HCG 23 and the galaxies HCG 22a, NGC 88 and NGC 89 are not included in the table because no Fabry-Perot data are 


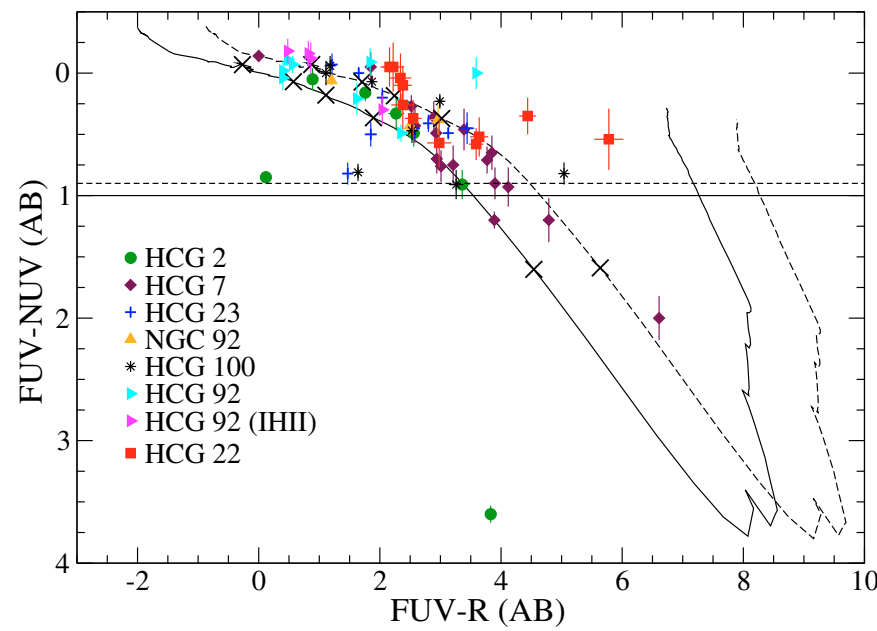

Fig. 18. GALEX FUV-NUV versus FUV-R of the intragroup objects. Models from Thilker et al. (2007) are shown as a solid line (no extinction correction) and dotted line (internal extinction of the Milky Way $E(B-V)=0.2)$. Crosses mark ages 10, 50, 100, 200, and $500 \mathrm{Myr}$, from the bottom right to the top left. We show only a few objects for HCG 92 due to the small field of view of the optical image compared with the GALEX image. Colors bluer than FUV-NUV $=0.9$ (dashed line) are typical of spiral and late-type galaxies (Gil de Paz et al. 2007). Objects bluer than FUV-NUV $=1$ (continuous line) were taken into account in our analysis.

available for these objects. The first four indicators for HCG 92 are also not included because no Fabry Perot velocity maps could be built given that for this group the ionized gas is outside the galaxies (Mendes de Oliveira et al. 2001; Plana et al. 1999).

By inspecting Table 11 one can see that three galaxies present disagreement between both sides of the rotation curves, HCG 2a, HCG 2b and NGC 92. These galaxies have close companions and they may have suffered a recent encounter. This has been modelled by Pedrosa et al. (2008) for major mergers and by Kronberger et al. (2006) for fly-bys and minor mergers. In the case of a major merger, Pedrosa et al. find that the significantly disturbed rotation curve occurs in a small time interval within $0.5 \mathrm{Gyr}$ of the occurrence of the pericenter. In the case of NGC 92, this time is consistent with the dynamical time of the tidal tail, $\sim 0.2 \mathrm{Gyr}$, as we derived from the maximum tidal extent divided by the maximum expected tidal velocity (Hibbard et al. 2005). At the tip of this tail, we detected a tidal dwarf galaxy candidate. Although the presence of double profiles in the central region of this galaxy could be associated with its LINER nucleus, double components are also observed along the tidal tail and these are not correlated to the $\mathrm{H} \alpha$ intensity. For these reasons we claim that the double components are most likely related to strong interactions. NGC 92, therefore, may have had a very recent interaction with one of its close neighbours. It is possible that HCG 2a and HCG $2 \mathrm{~b}$ also had a close encounter.

The kinematics of HCG $7 \mathrm{a}$ as a whole does not show signatures of strong interaction with other members of this group (although its velocity field is peculiar, as described in Sect. 4.1.2). No peculiarities were found in Mendes de Oliveira et al. (2003) for HCG 7c. Taking into account these kinematic results, members of HCG 7 have not had recent interactions with each other.

In the case of the triplet HCG 22, only one member, HCG 22c, has ionized gas and a symmetric rotation curve was determined. As we note in Sect. 4.1, HCG 22b has shells, which

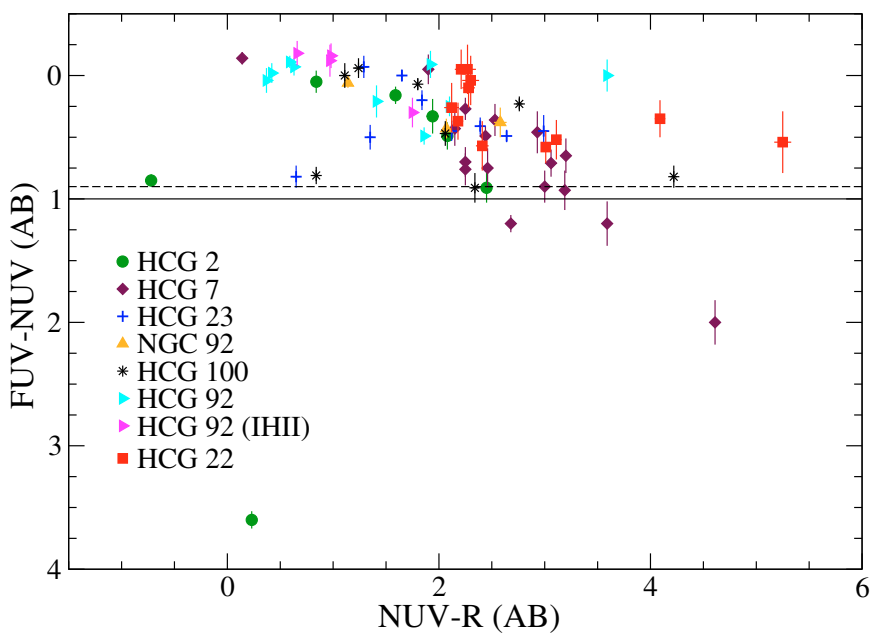

Fig. 19. FUV-NUV vs. NUV-R plot. See caption of Figs. 18.

indicates a possible merger between an elliptical and a small disk galaxy (Combes \& Charmandaris 1998). The timescale for shell formation is $1-10 \mathrm{Gyr}$.

Plana et al. (2003) showed that the members of group HCG 100 are in an advanced stage of interaction.

\subsection{Ultraviolet light as an evolutionary diagnostic}

Despite the fact that tidal dwarf galaxies have been found in a few interacting systems (e.g. Mendes Oliveira et al. 2001; Duc \& Mirabel 1998), other strongly interacting systems present no significant excess of dwarfs (Delgado-Donate et al. 2003). In Table 12, we present the confirmed intergalactic HII regions and TDGs taken from the literature. In our study, we found an excess of UV emitting regions in the field of HCG 92 and HCG 22. HCG 92 shows a bluer FUV-NUV color distribution compared with the control sample, which is in agreement with the previous studies for this group (Mendes de Oliveira et al. 2001, 2004). HCG 22, however, shows an intragroup color distribution similar to the control sample distribution, i.e. HCG 22 contains an older population of intragroup regions than HCG 92. It is possible that these regions are long-lived TDGs formed in an earlier interaction and they were able to survive. Similar results have been reported by Duc et al. (2007) for the Virgo cluster. This scenario is compatible with the studies developed by Bournaud \& Duc 2006. They found that at least $25 \%$ of the substructures located in tidal tails become long-lived bound objects that typically survive more than 2 Gyr. In the same way, Hunsberger et al. (1996) found that at least one-third of the dwarf galaxy population in compact groups is formed by galaxy interactions.

Although only HCG 92 and HCG 22 show higher field densities compared with their control samples, HCG 100 shows a blue peak in the FUV-NUV color distribution. This group contains TDGs (de Mello et al. 2008a; Urrutia-Viscarra et al. 2009) and has been suggested to be in an advanced evolutionary stage by Plana et al. (2003).

NGC 92 has only five intragroup regions, three of them (\#1, $\# 2$ and \#3) are located in the extended tidal tail of NGC 92 and are clearly seen in the NUV, FUV and $R$-band images. We note the presence of a strong UV emission close to the end of the tidal tail of NGC 92, similar to the UV emission from the Antennae TDG candidate (Hibbard et al. 2005). Our detected region at the end of the tidal tail is only 45 Myr old. Bournaud \& Duc (2006) performed simulations in order to relate TDGs and 
Table 11. Interaction indicators.

\begin{tabular}{|c|c|c|c|c|}
\hline \multirow[t]{2}{*}{ Interaction indicator $^{a}$} & \multicolumn{4}{|c|}{ Galaxy } \\
\hline & HCG 2a & HCG 2b & HCG 2c & \\
\hline Highly disturbed velocity field & + & - & - & \\
\hline Disagreement between both sides of the rotation curve & + & + & - & \\
\hline Gaseous versus stellar major-axis misalignment & $++^{b}$ & - & - & \\
\hline Changing position angle along major axis & + & - & - & \\
\hline Multiple components in the profiles & - & - & - & \\
\hline Tidal tails & - & - & - & \\
\hline High IR luminosity & - & + & - & \\
\hline \multirow[t]{2}{*}{ Central activity } & + & + & $\ldots$ & \\
\hline & HCG 7a & HCG 7b & HCG 7c & HCG 7d \\
\hline Highly disturbed velocity field & + & $\ldots$ & - & - \\
\hline Disagreement between both sides of the rotation curve & - & $\ldots$ & - & - \\
\hline Gaseous versus stellar major-axis misalignment & - & $\ldots$ & $\ldots$ & $+^{b}$ \\
\hline Changing position angle along major axis & - & $\ldots$ & $\ldots$ & - \\
\hline Multiple components in the profiles & - & $\ldots$ & $\ldots$ & - \\
\hline Tidal tails & - & - & - & - \\
\hline High IR luminosity & + & - & + & - \\
\hline \multirow{2}{*}{ Central activity } & - & $\ldots$ & $\ldots$ & $\ldots$ \\
\hline & HCG 22a & HCG 22b & HCG 22c & \\
\hline Highly disturbed velocity field & $\ldots$ & $\ldots$ & - & \\
\hline Disagreement between both sides of the rotation curve & $\ldots$ & $\ldots$ & - & \\
\hline Gaseous versus stellar major-axis misalignment & $\ldots$ & $\ldots$ & $+^{b}$ & \\
\hline Changing position angle along major axis & $\ldots$ & $\ldots$ & - & \\
\hline Multiple components in the profiles & $\ldots$ & $\ldots$ & - & \\
\hline Tidal tails & - & - & - & \\
\hline High IR luminosity & - & - & - & \\
\hline \multirow{2}{*}{ Central activity } & + & - & - & \\
\hline & HCG 23a & HCG 23b & HCG 23c & HCG 23d \\
\hline Tidal tails & - & - & - & $\ldots$ \\
\hline High IR luminosity & $\ldots$ & + & $\ldots$ & + \\
\hline \multirow[t]{2}{*}{ Central activity } & + & $\ldots$ & + & - \\
\hline & HCG 92b & HCG 92c & HCG 92d & HCG 92e \\
\hline Tidal tails & + & + & + & - \\
\hline High IR luminosity & + & + & - & - \\
\hline \multirow[t]{2}{*}{ Central activity } & - & + & - & - \\
\hline & HCG $100 a^{c}$ & HCG $100 b^{c}$ & HCG $100 \mathrm{c}^{c}$ & HCG $100 d^{c}$ \\
\hline Highly disturbed velocity field & + & + & + & + \\
\hline Disagreement between both sides of the rotation curve & $\ldots$ & $\ldots$ & $\ldots$ & $\ldots$ \\
\hline Gaseous versus stellar major-axis misalignment & - & + & + & - \\
\hline Changing position angle along major axis & + & + & + & - \\
\hline Multiple components in the profiles & - & - & - & - \\
\hline Tidal tails & - & + & - & - \\
\hline High IR luminosity & + & - & - & - \\
\hline \multirow[t]{2}{*}{ Central activity } & - & - & - & - \\
\hline & NGC 92 & NGC 89 & NGC 88 & NGC 87 \\
\hline Highly disturbed velocity field & - & $\ldots$ & $\ldots$ & $\ldots$ \\
\hline Disagreement between both sides of the rotation curve & + & $\ldots$ & $\ldots$ & $\ldots$ \\
\hline Gaseous versus stellar major-axis misalignment & - & $\ldots$ & $\ldots$ & $\ldots$ \\
\hline Changing position angle along major axis & + & $\ldots$ & $\ldots$ & $\ldots$ \\
\hline Multiple components in the profiles & + & $\ldots$ & $\ldots$ & $\ldots$ \\
\hline Tidal tails & + & - & - & - \\
\hline High IR luminosity & $\ldots$ & $\ldots$ & $\ldots$ & $\ldots$ \\
\hline Central activity & + & + & + & - \\
\hline
\end{tabular}

${ }^{a}$ References. - The indicator "High IR luminosity" was taken from the following sources: Allam et al. (1996), Verdes-Montenegro et al. (1998) and Gallagher et al. (2008). The indicator "Central Activity" was taken from: Shimada et al. (2000), de Carvalho et al. (1997), Coziol et al. (1998, 2000) and $\mathrm{Xu}$ et al. (2003).

${ }^{b}$ The disagreement between optical and kinematic position angle could be associated with the presence of a bar.

${ }^{c}$ From Plana et al. (2003).

satellite galaxies. They found that the most massive objects located at the end of tidal tails are likely to be the TDG progenitors. Therefore, considering its location and age, we classify the object in the tidal tail of NGC 92 as a TDG candidate. We also note that this object is detected in $\mathrm{H} \alpha$ (Fabry-Perot image), confirming its group membership. As has been noted for the Antennae (Hibbard et al. 2005), we detect a gradient in the colors of the regions detected along the tail. The bluest region is the TDG candidate, at the tip of the tail of NGC 92. 
Table 12. Other intergalactic HII regions and TDGs listed in the literature.

\begin{tabular}{|c|c|c|c|c|c|c|c|c|}
\hline System & ID & $M_{B}$ & $\begin{array}{l}\text { Velocity } \\
\left(\mathrm{km} \mathrm{s}^{-1}\right) \\
\end{array}$ & $12+\log (\mathrm{O} / \mathrm{H})$ & $\begin{array}{l}\text { Mass } \\
\left(M_{\odot}\right) \\
\end{array}$ & $\begin{array}{c}\text { SFR } \\
M_{\odot} \mathrm{yr}^{-1} \\
\end{array}$ & IHII-TDG & 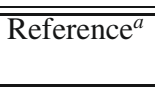 \\
\hline HCG 92 & $\mathrm{a}$ & -11.9 & 6600 & 8.48 & $2.0 \times 10^{4}$ & & IHII & 1 \\
\hline HCG 92 & $\mathrm{~b}$ & -12.1 & 6651 & 8.65 & $1.4 \times 10^{4}$ & & IHII & 1 \\
\hline HCG 92 & $\mathrm{c}$ & -12.5 & 6565 & 8.64 & $4.4 \times 10^{4}$ & & IHII & 1 \\
\hline HCG 92 & $\mathrm{~d}$ & -12.3 & 6616 & 8.53 & $3.6 \times 10^{4}$ & & IHII & 1 \\
\hline HCG 92 & 2 & -14.0 & & & $38 \times 10^{8}$ & 4.26 & TDG & 2 \\
\hline HCG 92 & 3 & -14.7 & & & & $\ldots$ & TDG & 2 \\
\hline HCG 92 & 4 & -13.4 & & & & $\ldots$ & TDG & 2 \\
\hline HCG 92 & 5 & -15.3 & & & & $\ldots$ & TDG & 2 \\
\hline HCG 92 & 6 & -15.0 & & & $20 \times 10^{8}$ & $\ldots$ & TDG & 2 \\
\hline HCG 92 & 8 & -14.2 & & & $138 \times 10^{8}$ & 0.38 & TDG & 2 \\
\hline HCG 92 & 20 & -12.6 & & & $6.7 \times 10^{8}$ & 0.45 & TDG & 2 \\
\hline HCG 92 & 21 & -14.4 & & & $24 \times 10^{8}$ & 0.73 & TDG & 2 \\
\hline HCG 92 & 22 & -14.2 & & & $4.3 \times 10^{8}$ & 0.63 & TDG & 2 \\
\hline HCG 92 & 23 & -13.0 & & & $2.4 \times 10^{8}$ & 0.20 & TDG & 2 \\
\hline HCG 16 & 1 & & 3634 & & & & IHII & 3 \\
\hline ESO 154-G023 & 1 & & $\ldots$ & & & & IHII & 3 \\
\hline NGC 1314 & 1 & & $\ldots$ & & & & IHII & 3 \\
\hline NGC 1533 & 1 & & 846 & & & & IHII & 3 \\
\hline NGC 1533 & 2 & & 831 & & & & IHII & 3 \\
\hline NGC 1533 & 3 & & $\ldots$ & & & & IHII & 3 \\
\hline NGC 1533 & 4 & & $\ldots$ & & & & IHII & 3 \\
\hline NGC 1533 & 5 & & 901 & & & & IHII & 3 \\
\hline IC 5052 & 1 & & $\ldots$ & & & & IHII & 3 \\
\hline IC 5052 & 2 & & $\ldots$ & & & & IHII & 3 \\
\hline ESO 238-G005 & 1 & & $\ldots$ & & & & IHII & 3 \\
\hline ESO 238-G005 & 2 & & $\ldots$ & & & & IHII & 3 \\
\hline ESO 149-G003 & 1 & & 949 & & & & IHII & 3 \\
\hline NGC 5291 & $\mathrm{a}$ & -15.54 & 4642 & 8.46 & $0.91 \times 10^{9}$ & 0.031 & TDG & 4 \\
\hline NGC 5291 & $\mathrm{~b}$ & -14.13 & 4536 & 8.39 & $0.13 \times 10^{9}$ & 0.004 & TDG & 4 \\
\hline NGC 5291 & $\mathrm{c}$ & -12.13 & 4617 & 8.57 & $\ldots$ & 0.002 & TDG & 4 \\
\hline NGC 5291 & d & -13.83 & 4571 & 8.41 & $0.54 \times 10^{9}$ & 0.001 & TDG & 4 \\
\hline NGC 5291 & e & -14.57 & 4415 & 8.57 & $0.43 \times 10^{9}$ & 0.002 & TDG & 4 \\
\hline NGC 5291 & f & -13.83 & 4035 & 8.63 & $\ldots$ & 0.002 & TDG & 4 \\
\hline NGC 5291 & g & -14.16 & 4162 & 8.47 & $\ldots$ & 0.012 & TDG & 4 \\
\hline NGC 5291 & $\mathrm{~h}$ & -13.98 & 4098 & 8.46 & $\ldots$ & 0.005 & TDG & 4 \\
\hline NGC 5291 & $\mathrm{i}$ & -16.38 & 3996 & 8.38 & $2.48 \times 10^{9}$ & 0.063 & TDG & 4 \\
\hline NGC 5291 & $\mathrm{j}$ & -13.74 & 4075 & 8.48 & $\ldots$ & 0.007 & TDG & 4 \\
\hline NGC 5291 & $\mathrm{k}$ & .13 .40 & 4041 & 8.45 & $\ldots$ & 0.002 & TDG & 4 \\
\hline
\end{tabular}

${ }^{a}$ References. 1: Mendes de Oliveira et al. (2004); 2: Mendes de Oliveira et al. (2001); 3: Ryan-Weber et al. (2004); 4: Duc \& Mirabel (1998). The mass for the candidates in NGC 5291 correspond to the HI mass.

HCG 2 and HCG 23 have no excess of UV regions in their field and have color distributions similar to their control sample. Although the intragroup regions in HCG 7 and in the CS have different color distribution (KS test, Table 10), it does not present an excess of intragroup regions in the field and moreover the regions in HCG 7 are generally redder than the colours of the CS regions. We conclude that these groups have not actively formed new regions in the intragroup medium, which might be an indication that they are in an early stage of evolution, i.e. that HCG 2, HCG 7 and 23 are non-evolved groups with respect to the UV properties.

We have also found that HCG 92 and HCG 100 intragroup regions have color distributions significantly different from those for other groups. We used the Gil de Paz et al. (2007) relation between the FUV-NUV color of a galaxy and its morphological type to evaluate the properties of their intragroup regions. Using Eq. (5) of Gil de Paz et al. (2007), objects bluer than FUV-NUV $=0.23$ are typically found to be irregular/compact galaxies (morphological type $T \geq 9.5$ ). Following this criterios, most of the objects in HCG 92 and in the blue peak of HCG 100 would have this morphological type, which is expected if some of them are TDG candidates.

What are the effects of dust and the typical ages of these UV-selected intragroup regions? The relation between UV and FIR emission can be used to obtain an estimate of the dust attenuation (Buat et al. 2005, and references therein). In our sample, we found that several star forming regions show blue FUV-NUV colors, such as those for the IHII regions in HCG 92 (Mendes de Oliveira et al. 2004). If the color of IHII is typical of star-forming regions, the attenuation in those objects is modest, with values lower than $A_{\mathrm{FUV}} \sim 1$ (see e.g. the extinction laws in Fig. 8b of Gil de Paz et al. 2007). Even though the FUV attenuation is low for this type of object, they must be corrected for this effect. The SFR that we calculated (see Table 9) using UV colors corrected for dust attenuation are modest but similar to the values found by Duc \& Mirabel (1998) for some TDG candidates (see Table 12).

We estimated ages using UV colors and the Milky Way extinction law (Thilker et al. 2007) only for the bluest objects (FUV-NUV < 0.1) in our sample (Tables 3-9). We compared 
the age values we estimated with the ones independently estimated from spectroscopy by Mendes de Oliveira et al. (2004, regions \#4, \#6 and \#8 in our Table 7) and found an excellent agreement in three of the four regions, showing ages $<10 \mathrm{Myr}$. The other IHII region (\#11 in Table 7) has FUV-NUV $\sim 0.3$ which is redder than FUV-NUV $<0.1$, the limit used to estimate ages using a Milky Way extinction law.

\subsection{The HI gas}

It is well known that interactions can cause severe disruption in the HI distribution and produce HI tidal tails which might host TDGs (Neff et al. 2005). Moreover, there are cases where $\mathrm{HI}$ is also found within clouds in the intergalactic medium (e.g. Williams et al. 2001) which were removed from galaxies' disks by tidal forces. Therefore, HI morphology and content may be a trace of the evolutionary stage of interacting systems. In order to quantify this effect, Verdes-Montenegro et al. (2001) analyzed the HI content of 72 compact groups of galaxies. They defined the $\mathrm{HI}$ deficiency in a group as $\operatorname{Def}_{\mathrm{HI}}=\log \left[M(\mathrm{HI})_{\text {pred }}\right]-$ $\log \left[M(\mathrm{HI})_{\text {obs }}\right]$, where the predicted mass for the group is the sum of the expected mass for each galaxy member. They proposed the following evolutionary sequence. "Phase 1", when the HI distribution in groups is relatively not perturbed, and almost all the $\mathrm{HI}$ gas is located in the disk of the galaxies with the remaining gas found in incipient tidal tails; "Phase 2", when 30 to $60 \%$ of the total HI mass forms tidal features; "Phase 3a", when the HI is located in tidal tails or when there is no HI; and "Phase 3b", when the HI gas is located in a large cloud encompassing all galaxies.

We obtained HI information for each group in our sample from the literature and evaluated their properties taking into account the Verdes-Montenegro et al. (2001) scenario as described below.

HCG 2 shows no HI deficiency, $\operatorname{Def}_{\mathrm{HI}}=0$, and the only interaction signature is the incipient $\mathrm{HI}$ tails arising from two of its members. For HCG 7, on the other hand, $\sim 80 \%$ of the expected HI is missing (Verdes-Montenegro et al. 2001).

For HCG 22, 72\% of the expected HI is missing (Verdes-Montenegro et al. 2001) and only HCG 22c shows HI emission. Price et al. (2000) noted that the strongest concentration of $\mathrm{HI}$ in the distribution is on the side towards the elliptical galaxy, although there are no obvious tidal tail features.

HCG 23 is classified as a group in "Phase 1" by Verdes-Montenegro et al. (2001). The observed and expected $\mathrm{HI}$ content is the same. Most of the HI in this group is associated with individual galaxies, as noted by Williams \& van Gorkom (1995). In the same study, they noted five additional members that lie outside the compact group. Velocity maps for galaxies a, $\mathrm{b}$ and $\mathrm{d}$ show an asymmetry in the motion of the gas with respect to the optical center. They suggest that this group is part of a much larger system.

The well studied HCG 92 shows two galaxies with the highest HI deficiency in the Verdes-Montenegro's sample: HCG 92b and HCG 92c. This group is classified as being in "Phase $3 \mathrm{a}$ " and shows most of the HI ( $\sim 33 \%$ of the expected $\mathrm{HI})$ in intergalactic clouds and tidal tails.

de Mello et al. (2008a) and Urrutia-Viscarra et al. (2009) studied HCG 100 and detected TDG candidates in the extended tidal tail visible only in HI and not in the optical. In this group only $32 \%$ of the expected HI is detected (Verdes-Montenegro et al. 2001).

Pompei et al. (2007) classified NGC 92 as a group in "Phase $3 b$ ". Only $32 \%$ of the expected HI was observed in this
Table 13. Evolutionary stages for the compact groups.

\begin{tabular}{cccc}
\hline \hline Group & Velocity field & HI & FUV-NUV \\
\hline HCG 2 & $+/-$ & - & - \\
HCG 7 & - & + & - \\
HCG 22 & - & + & + \\
HCG 23 & $\ldots$ & - & - \\
HCG 92 & + & + & + \\
HCG 100 & + & + & + \\
NGC 92 & + & + & + \\
\hline
\end{tabular}

For each interaction tracer, symbol + represents a more evolved group. Symbol - means a less evolved group. The + sign in the FUV-NUV column of NGC 92 refers to the blue color of the candiate TDG.

group $\left(\operatorname{Def}_{H I}=0.51\right)$. An extended HI tail follows the opti$\mathrm{cal} / \mathrm{UV}$ tail.

From the HI properties for each group, we conclude that galaxies in HCG 2 and HCG 23 do not show clear signatures of interaction. On the other hand, HCG 92 and HCG 100 show large tidal tails of neutral gas, far away from the main body of the galaxies.

\subsection{Evolutionary stage of each group}

In Table 11 we list some of the main interaction indicators for each galaxy and in Table 13 we summarize the signatures of evolution for each group using UV, HI and kinematic information. For instance, groups with an excess of UV regions, disturbed velocity fields and HI outside galaxies were considered as highly evolved.

Although HCG 2 does not show an excess of intragroup regions nor a deficiency in the HI content, the velocity field of HCG 2a shows several signatures of interactions, which may be produced by an interaction with its companion HCG $2 \mathrm{~b}$.

HCG 23 shows no UV excess and no HI gas in the intragroup medium, suggesting that this group is in an early-stage of evolution.

On the other hand, HCG 92 presents an evolved evolutionary stage in the UV analysis, with several star-forming regions in the intragroup medium confirmed spectroscopically (Mendes de Oliveira et al. 2004). It also contains HI in the intragroup medium.

HCG 100 also shows an advanced evolutionary stage. Plana et al. (2003) found strong signatures of interaction in their galaxy members, de Mello et al. (2008a) found some TDG candidates in the HI tail of HCG 100.

Although only $\sim 20 \%$ of the expected HI is observed in HCG 7, there is no excess in the field density of this group nor is there a significantly blue distribution in its color FUV-NUV distribution or disturbed kinematics of their galaxy members. This result suggests that the missing $\mathrm{HI}$ has not been used to form stars in the intragroup medium. On the other hand, Verdes-Montenegro et al. (2001) noted that it is possible to explain the HI deficiency in compact groups by tidal removal of the outer HI disk in the galaxies, which can explain the deficiency of HI in HCG 7 without subsequent star formation.

The evolutionary stage of HCG 22 is not as clear as for the other targets. If the excess of regions in HCG 22 is the result of previous galaxy interactions or merger events, a fraction of the HI gas might have been converted into stars. This scenario is supported by the HI observations, since only $28 \%$ of the expected gas is observed in this group. However, this group contains an elliptical galaxy, HCG 22a, which contains no signatures of 
mergers (da Rocha et al. 2002; de Souza et al. 2004). Moreover, HCG 22b shows shell structures, associated with mergers events. Therefore, if the intragroup regions belong to HCG 22, we suggest that they are formed from galaxy interaction and/or a merger event.

In NGC 92 we did not find any excess of UV regions in their field. However, galaxy NGC 92 shows an extended tidal tail and both sides of the rotation curve do not match. A TDG candidate is clearly detected in our multiwavelength study, although no rotation has been measured from our Fabry Perot data. HI gas has been used up in the process of forming stars, which might be one of the reasons why $69 \%$ of the $\mathrm{HI}$ is missing in this group.

Finally, we compare our results with another tracer of evolution in groups: X-ray observations. In the X-ray Atlas of Groups of Galaxies, Mulchaey et al. (2003) showed that HCG 92 shows a clear diffuse emision (i.e. hot intragroup medium). On the other hand, HCG 2, HCG 22 and HCG 23 did not present hot intragroup medium (Mulchaey et al. 2003). Interestigly, the spiral rich group NGC 92 has a hot intragroup medium, as was reported by Trinchieri et al. (2008). These results of X-ray emission are in agreement with our clasification for the evolutionary stage of compact groups.

\section{Conclusions}

We present new ultraviolet and new Fabry-Perot data for a sample of 5 compact groups of galaxies. For each galaxy, we present UV images, $\mathrm{H} \alpha$ velocity field, $\mathrm{H} \alpha$ monochromatic images, velocity dispersion maps and rotation curves.

We classified the groups according to their evolutionary stages and in addition we searched for young star-forming region candidates in galaxies with tidal tails and in the intragroup medium of the compact groups. Dozens of regions with ages < few Myr were found. We concluded that HCG 7 and HCG 23 are non-evolved groups. HCG 2 and HCG 22 show limited signatures of interaction. For instance, HCG 2a has a non-symmetric rotation curve and HCG 22 has an excess in the field density of star-forming regions and shell structures. We therefore classified both groups as being in a mildly interacting stage of evolution. We conclude that three groups, HCG 92, HCG 100 and NGC 92, are in advanced stages of interaction, contain extended HI tails and harbor young star-forming regions and TDGs. We are in the process of obtaining spectroscopy of a sub-sample of the intragroup objects in order to confirm their group membership. Preliminary results confirm that at least two of the UV-bright sources of HCG 100 (as listed in Table 8) are in the same redshift as the group members (Urrutia-Viscarra et al. 2009).

Acknowledgements. We would like to thank the referee, Dr. Sonia Temporin, for very useful comments and suggestions which were very important in improving this paper. S.T.-F. acknowledges the financial support of FAPESP through the Doctoral position, under contract 2007/07973-3. C.M.d.O. acknowledges support from the Brazilian agencies FAPESP (projeto temático 2006/56213-9), CNPq and CAPES. D.F.d.M. acknowledges support from GALEX grant NNG06GG45G. H.P. acknowledges the financial support of CAPES through the Pos-Doctoral position, under contract 3656/08-0. GALEX is a NASA Small Explorer, launched in 2003 April. We gratefully acknowledge NASA's support for construction, operation, and science analysis for the GALEX mission, developed in cooperation with the Centre National d'Etudes Spatiales of France and the Korean Ministry of Science and Technology. This research has made use of the NASA/IPAC Extragalactic Database (NED) which is operated by the Jet Propulsion Laboratory, California Institute of Technology, under contract with the National Aeronautics and Space Administration. We also acknowledge the usage of the HyperLeda database (http://leda.univ-lyon1. $\mathrm{fr}$ ).

\section{References}

Allam, S., Assendorp, R., Longo, G., Braun, M., \& Richter, G. 1996, A\&AS, 117,39

Amram, P., Plana, H., Mendes de Oliveira, C., Balkowski, C., \& Boulesteix, J. 2003, A\&A, 402, 865

Amram, P., Mendes de Oliveira, C., Plana, H., Balkowski, C., \& Hernandez, O. 2007, A\&A, 471, 753

Bell, E. F., Wolf, C., Meisenheimer, K., et al. 2004, ApJ, 608, 752

Bertin, E., \& Arnouts, S. 1996, A\&AS, 117, 393

Boulesteix, J. 2002, ADHOCw reduction Package, Publication of Observatoire de Marseille, http://www . oamp. fr/adhoc/

Bournaud, F., \& Duc, P. A. 2006, A\&A, 456, 481

Brown, M. J. I., Dey, A., Jannuzi, B. T., et al. 2007, ApJ, 654, 858

Buat, V., Iglesias-Páramo, J., Seibert, M., et al. 2005, ApJ, 619, L51

Coziol, R., Ribeiro, A. L. B., de Carvalho, R. R., \& Capelato, H. V. 1998, ApJ, 493, 563

Coziol, R., Iovino, A., \& de Carvalho, R. R. 2000, AJ, 120, 47

Coziol, R., Brinks, E., \& Bravo-Alfaro, H. 2004, AJ, 128, 68

Daigle, O., Carignan, C., Hernandez, O., Chemin, L., \& Amram, P. 2006, MNRAS, 368, 1016

da Rocha, C., Mendes de Oliveira, C., Bolte, M., Ziegler, B. L., \& Puzia, T. H. 2002, AJ, 123, 690

de Carvalho, R. R., Ribeiro, A. L. B., Capelato, H. V., \& Zepf, S. E. 1997, ApJS, 110,1

Debattista, V. P., Mayer, L., Carollo, C. M., et al. 2006, ApJ, 645, 209

Delgado-Donate, E. J., Muñoz-Tuñón, C., Deeg, H. J., \& Iglesias-Páramo, J. 2003, A\&A, 402, 921

de Mello, D. F., Dahlen, T., Gardner, J. P., \& Grogin, N. A. 2006, AJ, 132, 2014 de Mello, D. F., Torres-Flores, S., \& Mendes de Oliveira, C. 2008a, AJ, 135, 319 de Mello, D. F., Smith, L. J., Sabbi, E., et al. 2008b, AJ, 135, 548 de Souza, R. E., Gadotti, D. A., dos Anjos, S. 2004, ApJS, 153, 411

de Vaucouleurs, G., de Vaucouleurs, A., Corwin, H. G., Jr., et al. 1991, Third Reference Catalogue of Bright Galaxies (New York: Springer)

Duc, P.-A., \& Mirabel, I. F. 1998, A\&A, 333, 813

Duc, P.-A., Braine, J., Lisenfeld, U., Brinks, E., \& Boquien, M. 2007, A\&A, 475, 187

Epinat, B., Amram, P., Marcelin, M., et al. 2008a, MNRAS, 388, 500 Epinat, B., Amram, P., \& Marcelin, M. 2008b, MNRAS, 390, 466

Gallagher, S. C., Johnson, K. E., Hornschemeier, A. E., Charlton, J. C., \& Hibbard J. E. 2008, ApJ, 673, 730

Gerin, M., Combes, F., \& Athanassoula, E. 1990, A\&A, 230, 37

Gil de Paz, A., Boissier, S., Madore, B. F., et al. 2007, ApJS, 173, 185

Hibbard, J. E., Bianchi, L., Thilker, D. A., et al. 2005, ApJ, 619, L87

Hickson, P. 1982, ApJ, 255, 382

Hickson, P. 1993, ApL\&C, 29, 1

Hickson, P., Mendes de Oliveira, C., Huchra, J. P., \& Palumbo, G. G. 1992, ApJ, 399,353

Hunsberger, S. D., Charlton, J. C., \& Zaritsky, D. 1996, ApJ, 462, 50

Iglesias-Páramo, J., Buat, V., Takeuchi, T. T., et al. 2006, ApJ, 164, 38

Iovino, A. 2002, AJ, 124, 2471

Johnson, K., Hibbard, J., Gallagher, C., et al. 2007, AJ, 134, 1522

Kronberger, T., Kapferer, W., Schindler, S., et al. 2006, A\&A, 458, 69

Lupton 2005, SDSS website

Mendes de Oliveira, C. 1992, Ph.D. Thesis, British Columbia Univ.

Mendes de Oliveira, C., \& Hickson, P. 1994, ApJ, 427, 684

Mendes de Oliveira, C., Plana, H., Amram, P., Balkowski, C., \& Boulesteix, J. 1998, ApJ, 507, 691

Mendes de Oliveira, C., Plana, H., Amram, P., Balkowski, C., \& Bolte, M. 2001, AJ, 121, 2524

Mendes de Oliveira, C., Cypriano, E. S., Sodré, L., Jr., \& Balkowski, C. 2004, ApJ, L17

Mendes de Oliveira, C., Temporin, S., Cypriano, E. S., et al. 2006, AJ, 132, 570

Mulchaey, J. S., Davis, D. S., Mushotzky, R. F., \& Burstein, D. 2003, ApJS, 145, 39

Neff, S. G., Thilker, D. A., Seibert, M., et al. 2005, ApJ, 619, L91

Paturel, G., Petit, C., Prugniel, P., et al. 2003, A\&A, 412, 45

Pedrosa, S., Tissera, P. B., Fuentes-Carrera, I., \& Mendes de Oliveira, C. 2008, A\&A, 484, 299

Plana, H., Mendes de Oliveira, C., Amram, P., et al. 1999, ApJ, 516, L69

Plana, H., Amram, P., Mendes de Oliveira, C., Balkowski, C., \& Boulesteix, J. 2003, AJ, 125, 1736

Pompei, E., Dahlem, M., \& Iovino, A. 2007, A\&A, 473, 399

Price, R. M., Babic, B., \& Jones, K. 2000, ASPC, 209, 163

Prugniel, P., \& Heraudeau, P. 1998, A\&AS, 128, 299

Rose, J. A. 1977, ApJ, 211, 311

Rose, J. A. 1979, ApJ, 231, 10 
Ryan-Weber, E. V., Meuerer, G. R., Freeman, K. C., Putman, M. E., \& Webster, R. L. 2004, AJ, 127, 1431

Rubin, V. C., Hunter, D. A., \& Ford, W. K., Jr. 1991, ApJS, 76, 153

Salim, S., Rich, R. M., Charlot, S., et al. 2007, ApJS, 173, 267

Schelegel, D. J., Finkbeiner, D. P., \& Davis, M. 1998, ApJ, 500, 525

Seibert, M., Martin, D. C., Heckman, T. M., et al. 2005, ApJ, 619, L55

Shimada, M., Ohyama, Y., Nishiura, S., Murayama, T., \& Taniguchi, Y. 2000 AJ, 119, 2664

Temporin, S., Ciroi, S., Iovino, A., et al. 2005, ASSL, 329, 78

Torres-Flores, et al. 2009, in preparation

Thilker, D. A., Bianchi, L., Meurer, G., et al. 2007, ApJS, 173, 538

Treyer, M., Schiminovich, D., Johnson, B., et al. 2007, ApJS, 173, 256

Trinchieri, G., Iovino, A., Pompei, E., et al. 2008, A\&A, 484, 195
Tzanavaris, P., et al. 2009, in preparation

Urrutia-Viscarra, et al. 2009, in preparation

Verdes-Montenegro, L., Yun, M. S., Perea, J., del Olmo, A., \& Ho, P. T. P. 1998, ApJ, 497, 89

Verdes-Montenegro, L., Yun, M. S., Williams, B. A., et al. 2001, A\&A, 377, 812 Verdes-Montenegro, L., Del Olmo, A., Yun, M. S., \& Perea, J. 2005, A\&A, 430, 443

Williams, B. A., \& van Gorkom, J. H. 1995, ASPC, 70, 77

Williams, B. A., McMahon, P. M., \& van Gorkom, J. H. 1991, AJ, 101, 1957

Xu, C. K., Lu, N. Y., Condon, J. J., Dopita, M., \& Tuffs, R. J. 2003, ApJ, 595, 665

Xu, C. K., Iglesias-Páramo, J., Burgarella, D., et al. 2005, ApJ, 619L, 95

Zucca, E., Ilbert, O., Bardelli, S., et al. 2006, A\&A, 455, 879 